\title{
Drosophila DH31 Neuropeptide and PDF Receptor Regulate Night-Onset Temperature Preference
}

\author{
둘adahiro Goda, ${ }^{1}$ Xin Tang, ${ }^{1}$ Yujiro Umezaki, ${ }^{1}$ Michelle L. Chu, ${ }^{1}$ Michael Kunst, ${ }^{2}$ Michael N. Nitabach, ${ }^{2}$ and \\ (D) Fumika N. Hamada ${ }^{1,3,4,5}$ \\ ${ }^{1}$ Visual Systems Group, Abrahamson Pediatric Eye Institute, Division of Pediatric Ophthalmology, Cincinnati Children's Hospital Medical Center, \\ Cincinnati, Ohio 45229, ${ }^{2}$ Department of Cellular and Molecular Physiology, Department of Genetics Kavli Institute for Neuroscience, Yale University School \\ of Medicine, New Haven, Connecticut 06520, ${ }^{3}$ Division of Developmental Biology, Cincinnati Children's Hospital Medical Center, Cincinnati, Ohio 45229, \\ ${ }^{4}$ Japan Science and Technology Agency, Precursory Research for Embryonic Science and Technology (PRESTO), Saitama 332-0012, Japan, and 50partment \\ of Ophthalmology, College of Medicine, University of Cincinnati, Cincinnati, Ohio 45229
}

Body temperature exhibits rhythmic fluctuations over a $24 \mathrm{~h}$ period (Refinetti and Menaker, 1992) and decreases during the night, which is associated with sleep initiation (Gilbert et al., 2004; Kräuchi, 2007a,b). However, the underlying mechanism of this temperature decrease is largely unknown. We have previously shown that Drosophila exhibit a daily temperature preference rhythm (TPR), in which their preferred temperatures increase during the daytime and then decrease at the transition from day to night (night-onset) (Kaneko et al., 2012). Because Drosophila are small ectotherms, their body temperature is very close to that of the ambient temperature (Stevenson, 1985), suggesting that their TPR generates their body temperature rhythm. Here, we demonstrate that the neuropeptide diuretic hormone 31 (DH31) and pigmentdispersing factor receptor (PDFR) contribute to regulate the preferred temperature decrease at night-onset. We show that PDFR and tetheredDH31 expression in dorsal neurons 2 (DN2s) restore the preferred temperature decrease at night-onset, suggesting that DH31 acts on PDFR in DN2s. Notably, we previously showed that the molecular clock in DN2s is important for TPR. Although PDF (another ligand of PDFR) is a critical factor for locomotor activity rhythms, $P d f$ mutants exhibit normal preferred temperature decreases at night-onset. This suggests that DH31PDFR signaling specifically regulates a preferred temperature decrease at night-onset. Thus, we propose that night-onset TPR and locomotor activity rhythms are differentially controlled not only by clock neurons but also by neuropeptide signaling in the brain.

Key words: circadian rhythm; DH31; Drosophila; PDFR; temperature preference behavior; thermoregulation

\section{Significance Statement}

Body temperature rhythm (BTR) is fundamental for the maintenance of functions essential for homeostasis, such as generating metabolic energy and sleep. One major unsolved question is how body temperature decreases dramatically during the night. Previously, we demonstrated that a BTR-like mechanism, referred to as temperature preference rhythm (TPR), exists in Drosophila. Here, we demonstrate that the diuretic hormone 31 (DH31) neuropeptide and pigment-dispersing factor receptor (PDFR) regulate preferred temperature decreases at night-onset via dorsal neurons 2. This is the first in vivo evidence that DH31 could function as a ligand of PDFR. Although both DH31 and PDF are ligands of PDFR, we show that DH31 regulates night-onset TPR, but PDF does not, suggesting that night-onset TPR and locomotor activity rhythms are controlled by different neuropeptides via different clock cells.

\section{Introduction}

Body temperature rhythm (BTR) is fundamental for maintaining homeostasis, such as in generating metabolic energy and sleep

\footnotetext{
Received March 23, 2016; revised Aug. 24, 2016; accepted Sept. 22, 2016.

Author contributions: T.G. and F.N.H. designed research; T.G., X.T., Y.U., and M.L.C. performed research; M.K. and M.N.N. contributed unpublished reagents; T.G., X.T., Y.U., and F.N.H. analyzed data; T.G. and F.N.H. wrote the paper.

This work was supported by a Trustee Grant and Research Innovation/Pilot funding from Cincinnati Children's Hospital, Charlotte R. Schmidlapp Woman Scholars, Japan Science and Technology (JST)/Precursory Research for Embryonic Science and Technology (PRESTO), the March of Dimes, and National Institutes of Health R01 Grant GM107582 to F.N.H. We thank Dr. Patrick Emery for the CIk4.5F-Gal4 and CIk4.1M-Gal4 flies, Dr. Paul Taghert for the Pdf ${ }^{01}$, Pdfr ${ }^{5304}$, and UAS-Pdfr flies, Dr. Michael Nitabach for UAS-t-Pdf, UAS-t-Dh31, UAS-t-PDF-SCR flies and the anti-DH31 antibody, and the Bloomington Drosophila Stock Center for the fly lines. We thank Drs. Christian I. Hong, Emi Nagoshi, and Haruna Kaneko, and the Hamada laboratory members for their comments and advice on this manuscript, as well as Dr. Kazuhiko Kume for advice on sleep data analysis.

The authors declare no competing financial interests.
}

(Aschoff, 1983; Refinetti and Menaker, 1992; Kräuchi, 2002, 2007a,b; Gilbert et al., 2004; Weinert, 2010; Gerhart-Hines et al., 2013). BTR is one of the most robust circadian outputs and can affect the peripheral clocks of mammals (Brown et al., 2002; Buhr et al., 2010). The rhythmic patterns of BTR and locomotor activity rhythms are analogous. For instance, in diurnal mammals, both body temperature and locomotor activity increase during the daytime and decrease at night (Satlin et al., 1995; Weinert and

Correspondence should be addressed to Fumika N. Hamada, Visual Systems Group, Abrahamson Pediatric Eye Institute, Division of Pediatric Ophthalmology, Children's Hospital Medical Center, Cincinnati, $\mathrm{OH} 45229$. E-mail: fumika.hamada@cchmc.org

DO1:10.1523/JNEUROSCI.0964-16.2016

Copyright $\odot 2016$ the authors $\quad 0270-6474 / 16 / 3611739-16 \$ 15.00 / 0$ 
Waterhouse, 1998; Refinetti, 1999). Nonetheless, BTR and locomotor activity rhythms are regulated by different subsets of subparaventricular zone (SPZ) neurons, suggesting that these rhythms are controlled independently (Saper et al., 2005a,b).

Mammals are not the only examples of this phenomenon. We have previously shown that Drosophila exhibit a daily temperature preference rhythm (TPR), in which their preferred temperatures increase during the daytime and then decrease at the transition from day to night (night-onset) (Kaneko et al., 2012). Because Drosophila are small ectotherms, their body temperature is very close to that of the ambient temperature (Stevenson, 1985), suggesting that their TPR generates their BTR. In Drosophila, we showed that TPR and locomotor activity rhythms are regulated by different subsets of clock neurons (Kaneko et al., 2012). There are $\sim 150$ central pacemaker cells in the fly brain, which are functionally homologous to mammalian suprachiasmatic nucleus (SCN) neurons (Welsh et al., 2010). These pacemaker cells are approximately divided into lateral neurons [LNs; small ventral LNs (s-LNvs), large ventral LNs (l-LNvs), and dorsal lateral neurons (LNds)] and dorsal neurons (DNs; DN1, DN2, and DN3) based on their location and size (Dubruille and Emery, 2008). We previously found that the clocks in DN2s are critical for regulating TPR during the daytime (Kaneko et al., 2012), but not for regulating locomotor activity rhythms (Kaneko et al., 2012).

Neuropeptides and their receptors have important roles in synchronizing circadian clocks (Taghert and Nitabach, 2012). A class II G-protein-coupled receptor, pigment-dispersing factor receptor (PDFR; Hyun et al., 2005; Lear et al., 2005; Mertens et al., 2005), and its ligand (PDF) play important roles in synchronizing circadian clocks and are required for robust circadian locomotor activity in Drosophila (Choi et al., 2009, 2012; Duvall and Taghert, 2012; Taghert and Nitabach, 2012). Notably, PDF and PDFR function in a similar manner to vasoactive intestinal peptide (VIP) and its receptor VPAC2 in mammals, both of which play important roles in the ability of clock neurons to regulate the rhythmicity and synchrony of both locomotor activity rhythms and BTRs (Harmar et al., 2002; Aton et al., 2005; Hannibal et al., 2011; Schroeder et al., 2011).

Recent reports have suggested that, in addition to PDF, diuretic hormone 31 (DH31) also activates PDFR based on in vitro experiments (Mertens et al., 2005) and a study that used brain imaging with bath-applied DH31 (Shafer et al., 2008). Moreover, it has been shown that DH31 is expressed in the posterior dorsal neurons 1 (DN1ps) and that it modulates sleep as a wakepromoting signal before dawn but does not affect locomotor activity rhythms in Drosophila (Kunst et al., 2014). DH31 is a functional homolog of mammalian calcitonin gene-related peptide (CGRP), which mediates thermosensation and thermoregulation (Coast et al., 2001; Johnson et al., 2005; Seybold, 2009; McCoy et al., 2013). However, it is unknown whether CGRP is involved in the regulation of BTR in mammals.

Here, we demonstrate that DH31 and PDFR play important roles for TPR at night-onset. DN2s are the main clock cells for TPR (Kaneko et al., 2012), and our data suggest that DH31 binding to PDFR in DN2s regulates temperature preference decreases at night-onset, which is the first in vivo evidence that DH31 could function as a ligand of PDFR. Therefore, we propose that circadian locomotor activity and night-onset TPR are regulated by different neuropeptides that use the same receptor expressed in different clock cells.

\section{Materials and Methods}

\section{Fly lines}

All the flies were raised in $12 \mathrm{~h}$ light/dark cycles at $25^{\circ} \mathrm{C}$; zeitgeber time (ZT) 0 is lights-on, ZT12 is lights-off. $w^{1118}$ flies were used for wild-type (WT) flies. Transgenic flies bearing membrane-tethered DH31 ( $t-D H 31$; UAS- $t-D H 31-M L: B 4$ ), membrane-tethered PDF (t-PDF; UAS- $t-P D F-$ $M L: M 2 a)$, or membrane-tethered SCR (UAS-t-PDF-SCR: $2 \mathrm{~A})$ were from Dr. Michael Nitabach (Choi et al., 2009). Pdfr ${ }^{5304}$, UAS-Pdfr were from Dr. Paul Taghert. $y w ; P d f^{01}, y w ; D h 31^{\# 51}$ flies (Head et al., 2015) and $P d f r^{5304}$ were backcrossed with $w^{1118}$ and written as $w ; P d f^{01}, w ; D h 31^{\# 51}$ and $P d f r^{5304-b c}$, respectively. tim-Gal4 (expressed in all clock neurons), Clk4.1M-Gal4 (expressed in $\sim 8-10$ DN1ps) and Clk4.5F-Gal4 (expressed in $\sim 4$ DN1ps; L. Zhang et al., 2010; Y. Zhang et al.), Clk9M-Gal4 (expressed in s-LNvs and DN2s) and Clk9M-Gal4, Pdf-Gal80 (expressed in DN2s; Kaneko et al., 2012) were used. Pdf-Gal80 was used to suppress the Clk9M-Gal4 expression in s-LNvs (Stoleru et al., 2005).

\section{Immunohistochemistry}

Immunostaining was performed as described previously (Hamada et al., 2008; Tang et al., 2013), with the following modifications: 5\% normal goat serum in PBST (PBS plus 0.3 or $0.5 \%$ Triton X-100) for blocking and antibody incubations. DH31 staining was performed with the guinea pig or rabbit anti-DH31 antibody (1:200; Kunst et al., 2014) and donkey anti-guinea pig or anti-rabbit Cy5 (1:200, Jackson ImmunoResearch). PDF staining was used with the mouse anti-PDF antibody or the guinea pig anti-VRI (VRILLE) antibody (Glossop et al., 2003; 1:200) and antimouse or guinea pig Alexa Fluor 647 (1:200; Jackson Immuno Research). Mounted brains were scanned using a Zeiss LSM5 Pascal confocal microscope. Images were digitally projected by $Z$-stacks.

\section{Temperature preference behavioral assay and data analysis}

Temperature preference behavioral (TPB) assay and data analysis were performed as described previously (Kaneko et al., 2012; Goda et al., 2014). The flies were raised at $25^{\circ} \mathrm{C}$ in $12 \mathrm{~h}$ light/dark (LD) conditions. The TPB assays were performed for $30 \mathrm{~min}$ in an environmental room maintained at $25^{\circ} \mathrm{C} / 65-70 \%$ relative humidity. Twenty to 30 flies were used for one trial and were never reused. Control and experimental trials were performed in parallel or independently. After the $30 \mathrm{~min}$ behavioral assay, the number of flies completely on the apparatus were counted. Flies partially or completely on the walls of the apparatus cover were not included in the data analysis. The percentage of flies within each $1^{\circ} \mathrm{C}$ temperature interval on the apparatus was calculated by dividing the number of flies within each $1^{\circ} \mathrm{C}$ interval by the total number of flies on the apparatus. The location of each $1^{\circ} \mathrm{C}$ interval was determined by measuring the temperature at six different points on the bottom of the apparatus. Data points were plotted as a percentage of flies within a $1^{\circ} \mathrm{C}$ temperature interval. The weighted mean of the preferred temperature was calculated by summing the products of the percentage of flies within a $1^{\circ} \mathrm{C}$ temperature interval and the corresponding temperature (e.g., fractional number of flies $\times 18.5^{\circ} \mathrm{C}+$ fractional number of flies $\times$ $19.5^{\circ} \mathrm{C}+\ldots$ fractional number of flies $\times 32.5^{\circ} \mathrm{C}$ ).

\section{TPR assay}

The TPR assays were performed for $30 \mathrm{~min}$ in light during the day and dark during the night and were tested $\geq 5$ times in each time zone (ZT1-ZT3, ZT4-ZT6, ZT7-ZT9, ZT10-ZT12, ZT13-ZT15, ZT16ZT18, ZT19-ZT21, and ZT22-ZT24). If the SEM of averaged preferred temperatures was not $<0.3$ after the five trials, we performed more trials until SEM reached $<0.3$. To have a full curve of $24 \mathrm{~h}, \geq 40$ experiments were necessary. In each time zone, the weighted mean preferred temperatures from each trial were averaged together and the SEM was calculated.

\section{Statistical analysis}

One-way ANOVA. To determine whether the datasets were of normal distribution, either Shapiro-Wilk normality test or Kolmogorov-Smirnov test was used. If it passed, the one-way ANOVA test and the TukeyKramer test were used. If it failed, Kruskal-Wallis test one-way ANOVA on ranks was used (see Tables 2, 3). 


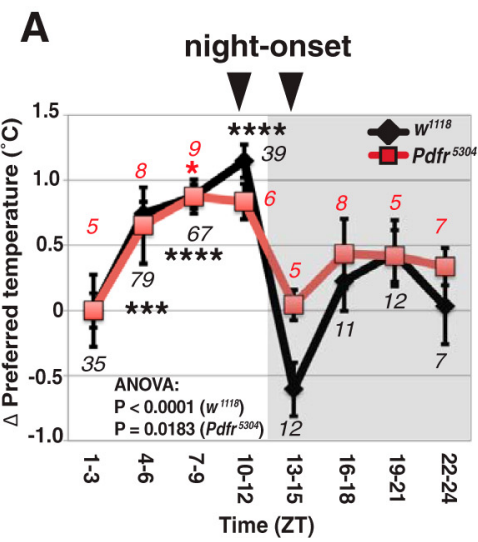

B daytime

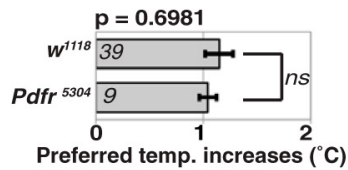

\section{C night-onset}

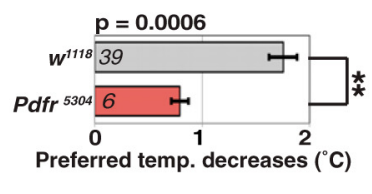

D night-onset

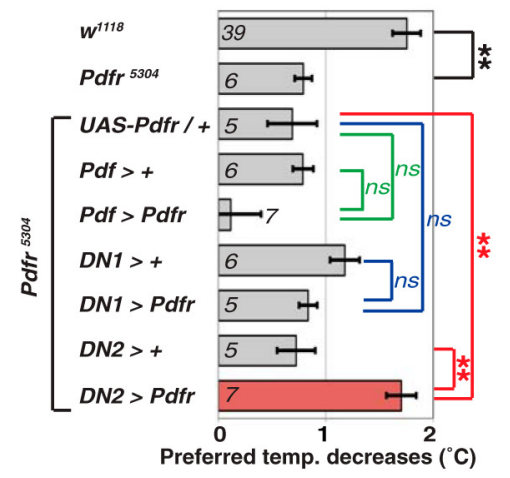

Figure 1. Pdfr is required for night-onset TPR. A, TPR phenotype of $w^{1118}$ (black line) and Pdfr ${ }^{5304}$ (red line) flies in LD cycles. The preferred temperatures at each time point were normalized by subtracting the preferred temperature at ZT1-ZT3 because $P d f r^{5304}$ exhibited a mild warm-temperature preference. The original data are shown in Figure $2 F$. Error bars indicate the SEM. Italicized numbers represent the number of assays (ZTO is lights-on, ZT12 is lights-off). Kruskal-Wallis test one-way ANOVA on ranks or one-way ANOVA: $p<0.0001\left(w^{1118}\right)$ and $p=0.0183\left(P d f{ }^{5304}\right)$. Post hoc test compared with ZT1-ZT3: ${ }^{* * *} p<0.0001,{ }^{* * *} p<0.001$, or ${ }^{*} p<0.05$. The detailed statistical analysis is shown in Table 3. $B$, Comparison of preferred temperature increases during the daytime (ZT1-ZT12) for $w^{1118}$ and Pdfr ${ }^{5304}$ flies. The values for temperature increases during the daytime were calculated by subtracting the preferred temperature at ZT1-ZT3 from the preferred temperature at ZT10-ZT12 $\left(w^{1118}\right)$ or ZT7-ZT9 $\left(P d f r^{5304}\right)$. $t$ test: $p=0.6981$. C, Comparison of preferred temperature decreases at night-onset (ZT10 -ZT12 to ZT13-ZT15) for $w^{1118}$ and Pdfr ${ }^{5304}$ flies. The values for temperature decreases at night-onset were calculated by subtracting the preferred temperature at ZT13-ZT15 from the preferred temperature at ZT10 -ZT12. $t$ test: $p=0.0006$. $D$, Comparison of preferred temperature decreases at night-onset (ZT10 -ZT12 to ZT13-ZT15) for Pdfr ${ }^{5304}$ rescue and their control flies. UAS-Pdfr was expressed in a subset of clock cells using the following Gal4 and Gal80 drivers: Pdf (Pdf-Gal4), DN1(Clk4.5F-Gal4) and DN2 (Clk9M-Gal4; Pdf-Gal80). One-way ANOVA and Tukey-Kramer test compared with controls: **p $<0.01$ (Table 2). Two-way ANOVA between controls and $P d f r$ rescue in DN2s flies showed a significant interaction between genotype and time $\left(F_{(2,28)}=3.627, p=0.0397 ;\right.$ Table 4).

Two-way ANOVA. To compare the differences between ZT10ZT12 and ZT13-ZT15 in control and experimental flies, we used two-way ANOVA particularly focusing on the interaction. Sidak's multiple-comparison test was used for Post hoc analysis (see Table 4).

\section{Behavioral analysis of locomotor activity and sleep}

Locomotor activity assay and data analysis were performed as described previously (Head et al., 2015; Umezaki et al., 2012). Flies were reared in $12 \mathrm{~h} \mathrm{LD}$ cycles at $25^{\circ} \mathrm{C}$. One-5-d-old male flies were used in locomotor activity experiments. Drosophila Activity Monitoring (DAM) systems (TriKinetics, http://www.trikinetics.com/) were placed in an incubator (MIR-154, Sanyo Scientific). Lights in the incubator (a $15 \mathrm{~W}$ cool-white fluorescent lamp; FL15D, Toshiba) were connected to an electric timer. Light intensity was $\sim 800$ lux. The locomotor activity was monitored in $12 \mathrm{~h} \mathrm{LD}$ cycles for $5 \mathrm{~d}$ and constant dark conditions for $>10 \mathrm{~d}$ at $25^{\circ} \mathrm{C}$. The free-running period was calculated by a $\chi^{2}$ periodogram (Sokolove and Bushell, 1978) with Actogram J software (Schmid et al., 2011). The power value was calculated according to the previous report (Umezaki et al., 2012). In sleep data analysis, sleep profiles were obtained using Microsoft Excel-based software as described by Tomita et al. (2011) and averaged across $4 \mathrm{~d}$.

\section{Results \\ PDFR is required for preferred temperature decreases at night-onset}

When WT flies are released onto a temperature gradient $\left(18-32^{\circ} \mathrm{C}\right)$, they prefer $\sim 25^{\circ} \mathrm{C}$ (Hamada et al., 2008). This preferred temperature increases during the daytime (ZT1-ZT12), decreases dramatically at night-onset (ZT10-ZT12 to ZT13-ZT15; Kaneko et al., 2012), and returns to $\sim 25^{\circ} \mathrm{C}$ before dawn (ZT22-ZT24).

To understand the molecular mechanisms of TPR, we first focused on the role of PDFR in TPR, because the PDFR is a neuropeptide receptor that functions to synchronize the circadian clock. We found that the preferred temperature of $P d f r^{5304}$ mutants increased during the daytime (Fig. $1 A, B$ ), but decreased only $\sim 0.8^{\circ} \mathrm{C}$ at night-onset (ZT10-ZT12 to ZT13-ZT15) while WT flies exhibited robust preferred temperature decreases of $\sim 1.5-2^{\circ} \mathrm{C}$ (Fig. $1 A, C$ ). The data suggest that PDFR is involved in regulating TPR at night-onset (ZT10-ZT15). Since WT flies dramatically decrease preferred temperature at night-onset, when many physiological changes presumably occur to maintain animal homeostasis, we sought to dissect the molecular mechanisms of night-onset TPR (ZT10-ZT12 to ZT13-ZT15) by focusing on the roles of PDFR.

\section{PDFR in DN2s is critical for night-onset TPR}

PDFR is expressed in a large subset of clock cells within the brain (Im and Taghert, 2010). To determine which clock neurons are important for night-onset TPR, we performed cDNA rescue experiments by expressing PDFR in a subset of the clock neurons. When PDFR was expressed in LNvs or DN1ps with Pdf-Gal4 or Clk4.5F-Gal4, respectively, PDFR expression did not sufficiently rescue the $P d f r^{5304}$ mutant phenotype (Fig. $1 D$, green and blue)

Next, we focused on DN2s because we have previously shown that DN2 function is important for preferred temperature increases during the daytime (Kaneko et al., 2012). Importantly, the previous data regarding PDFR-MYC expression and brain imaging with bath-applied PDF suggest that PDFR is expressed in DN2s (Shafer et al., 2008; Im and Taghert, 2010). As previously reported, Clk9MGal4; Pdf-Gal80 was only expressed in DN2s and not expressed in s-LNvs at $25^{\circ} \mathrm{C}$ (Kaneko et al., 2012; Fig. 2A,B). Therefore, we used Clk9M-Gal4; Pdf-Gal80 to express Pdfr in DN2s in the Pdfrr ${ }^{5304}$ background.

During the daytime, even though the total increases of preferred temperature of the Gal4 and UAS controls in the $P d f r^{5304}$ background were lower than that of the rescue flies (Fig. 2D; see Table 2), their preferred temperature still showed a statistically significant increase (Fig. $2 C$; see Table 3 ). At night-onset, the preferred temperature decreases of the rescue flies were restored (Figs. $1 D$, red, $2 C, E$ ) and statistically different from that of the Gal4 and UAS controls (see Tables 2, 3). These data indicate that PDFR expression in DN2s is sufficient for night-onset TPR.

It is worth noting that $P d f r^{5304}$ mutants preferred a slightly warmer temperature compared with WT and exhibited a phase-advanced phenotype with a peak at ZT7-ZT9 (Fig. 2F). 

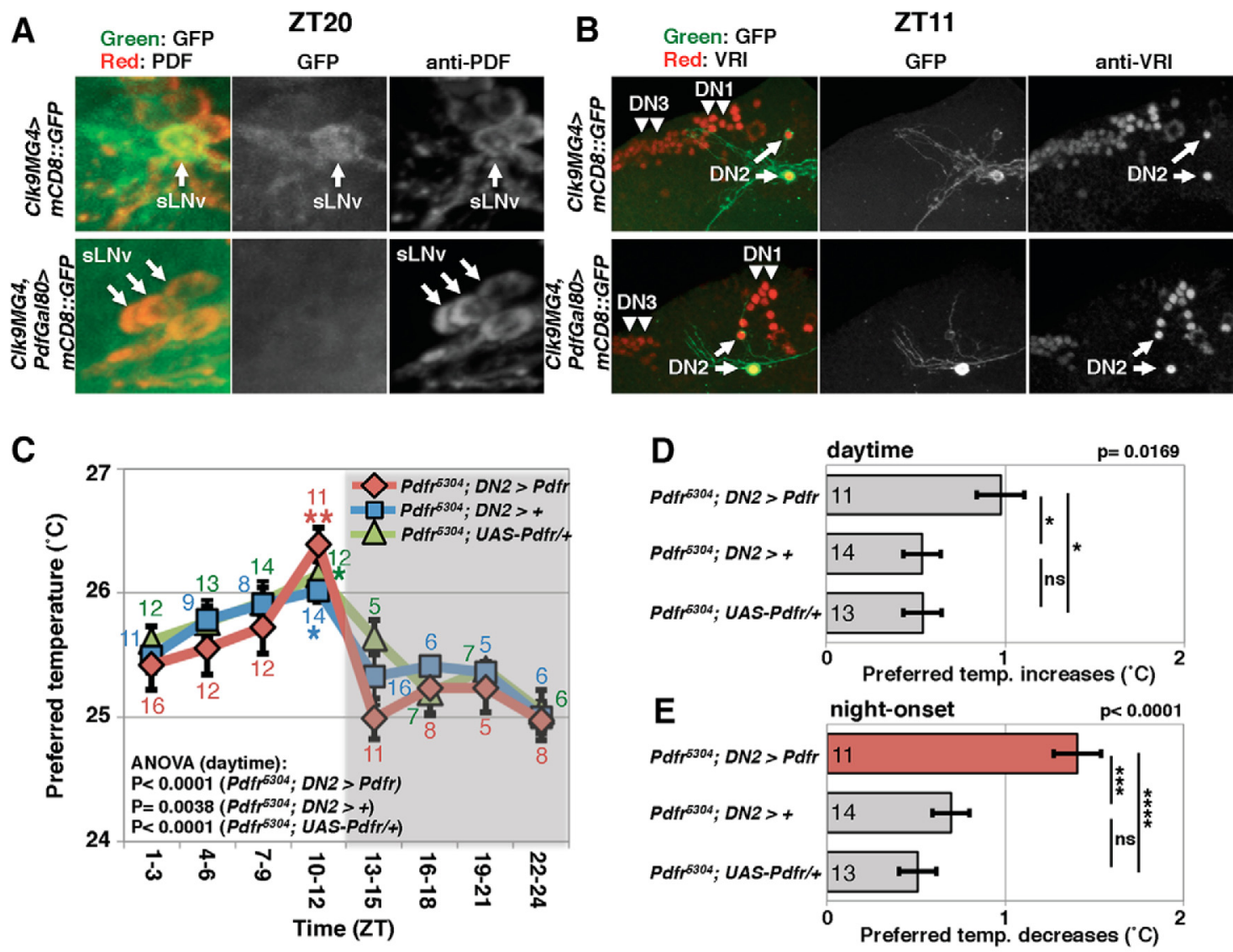

$\mathbf{F}$

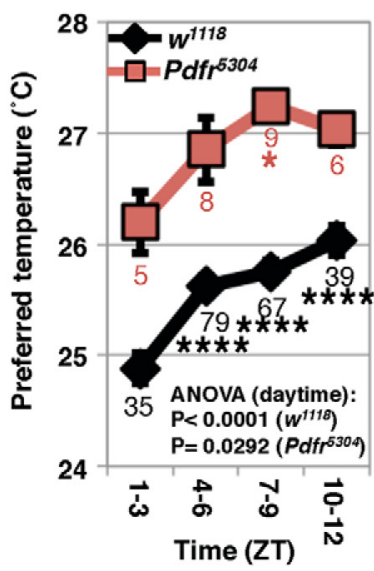

G

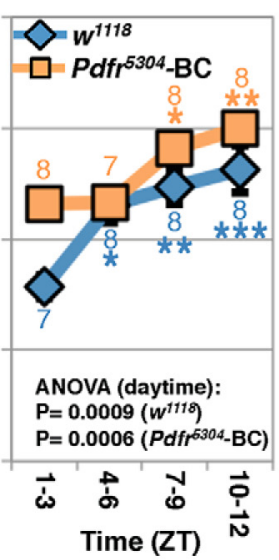

H

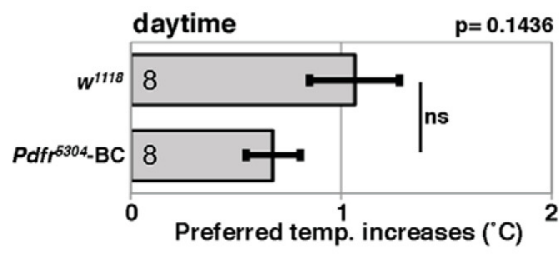

I

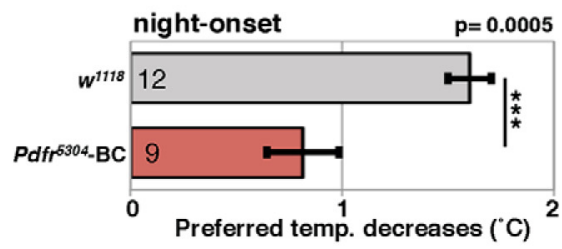

Figure 2. TPR phenotype of Pdfr mutant rescue flies and Pdfr mutant backcrossed flies. $\boldsymbol{A}, \boldsymbol{B}$, Immunostaining with anti-PDF $(\boldsymbol{A})$ or anti-VRI $(\boldsymbol{B})$ on the fly brain of $C l k 9 M$-Gal $4>$ UAS$m C D 8:: G F P$ and CIk9M-Gal4, Pdf-Gal80> UAS-mCD8::GFP flies at $25^{\circ} \mathrm{C}$. These images are the confocal stacks. GFP signals are shown in green. PDF $(\boldsymbol{A})$ or VRI $(\boldsymbol{B})$ signals are shown in red. Clk9M-Gal4, Pdf-Gal80 was not expressed in s-LNv (A). GFP was expressed strongly in only one of the two DN2s in $\sim 80 \%$ of Clk9M-Gal4; Pdf-Gal80 and Clk9M-Gal4 brains (B). C, A $24 \mathrm{~h}$ profile of the TPR phenotype of $P d f{ }^{5304}$ rescue flies in LD cycles. UAS-Pdfr was expressed in DN2 cells using CIk9M-Gal4, Pdf-Gal80. Error bars indicate SEM. Italicized numbers represent the number of assays. Kruskal-Wallis test one-way ANOVA on ranks or one-way ANOVA and post hoc test compared TPB using ZT1-ZT3 as the standard: ${ }^{* *} p<0.01$ or ${ }^{*} p<0.05$ (Table 3). $\boldsymbol{D}$, Comparison of preferred temperature increases during the daytime (ZT1-ZT12) for Pdfr rescue in DN2s. Error bars indicate the SEM. One-way ANOVA and Tukey-Kramer test were used to compare with each control: ${ }^{*} p<0.05$ (Table 2). $\boldsymbol{E}$, Comparison of preferred temperature decreases at night-onset (ZT10-ZT12 to ZT13-ZT15) for Pdfr rescue in DN2s. Error bars indicate the SEM. One-way ANOVA and Tukey-Kramer test were used to compare against each control: ${ }^{* * * *} p<0.0001$ or ${ }^{* * *} p<0.001$ (Table 2). $\boldsymbol{F}$, TPR phenotype of $w^{1118}$ (black line) and $P d f r^{5304}$ (red line) flies in LD cycles. This was the original data of Figure $1 A$ before normalizing. Pdfr ${ }^{5304}$ exhibited a slightly warmer temperature preference than that of WT and an advanced TPR, where the peak was at ZT7-ZT9. G, TPR phenotype of $w^{1118}$ (blue line) and Pdfr ${ }^{5304-B C}$ (orange line) flies in LD cycles. Because different apparatuses were used for $\boldsymbol{F}$ and $\boldsymbol{G}$, the preferred temperatures of WT $\left(\boldsymbol{F}\right.$, black; $\boldsymbol{G}$, blue) were slightly different. Importantly, WT flies using either apparatus increased $\sim 1^{\circ} \mathrm{C}$ preferred temperature during the daytime, showing normal TPR (Table 3). Pdfr ${ }^{5304-B C}$ exhibited a similar TPB as WT. H, Comparison of preferred temperature increases during the daytime (ZT1-ZT12) for w ${ }^{1118}$ and Pdff ${ }^{504-B C}$ flies. The values for temperature increases during the daytime were calculated by subtracting the preferred temperature at ZT1-ZT3 from the preferred temperature at ZT10 -ZT12. $t$ test: $p=$ 0.1436. I, Comparison of preferred temperature decreases at night-onset (ZT10 -ZT12 to ZT13-ZT15) for $w^{1118}$ and Pdfr ${ }^{5304-B C}$ flies. The values for temperature decreases at night-onset were calculated by subtracting the preferred temperature at ZT13-ZT15 from the preferred temperature at ZT10-ZT12. $t$ test: $p=0.0005$. 
A

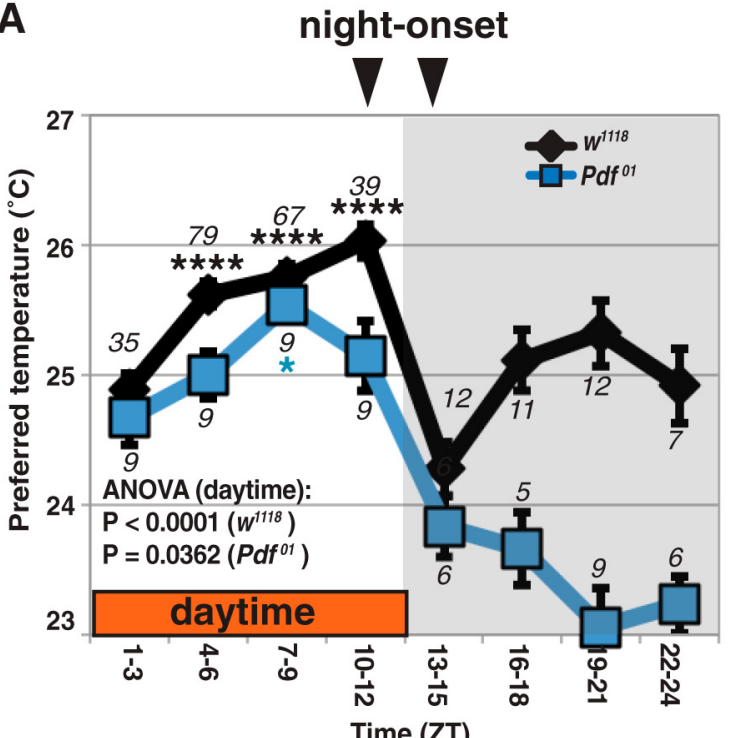

D

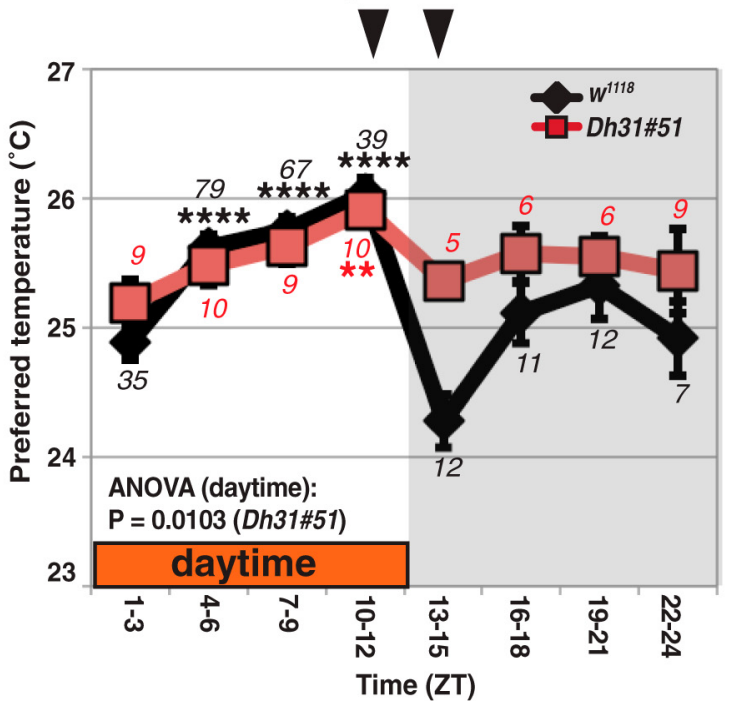

B

\section{daytime}

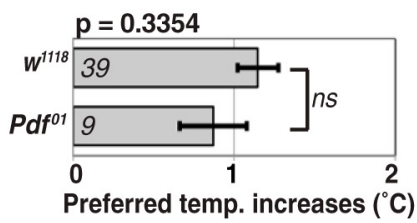

C

night-onset

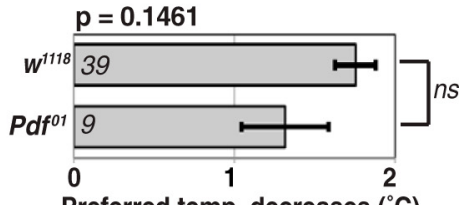

E

\section{daytime}

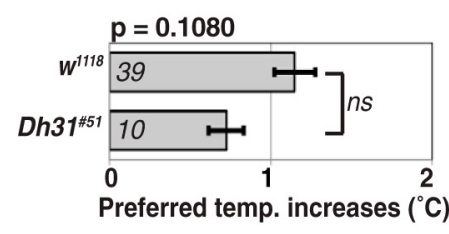

F

night-onset

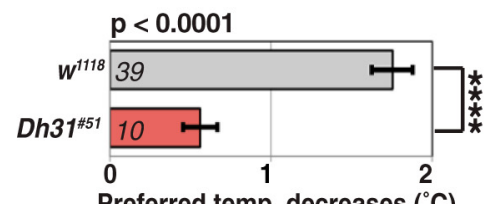

Figure 3. DH31 but not PDF is required for night-onset TPR. $A$, TPR phenotype of $w^{1118}$ (black line) and Pdf ${ }^{01}$ (blue line) flies in LD cycles. Error bars indicate SEM. Italicized numbers represent the number of assays. One-way ANOVA: $p<0.0001\left(w^{1118}\right)$ and $p=0.0362\left(P d f^{01} ; F_{(3.32)}=3.205\right)$. Tukey-Kramer test compared with ZT1-ZT3: ${ }^{* * * *} p<0.0001$ or ${ }^{*} p<0.05$ in Table $3 . \boldsymbol{B}_{\text {, }}$ Comparison of preferred temperature increases during the daytime (ZT1-ZT12) for $w^{1718}$ and $P d f^{01}$ flies. The values for temperature increases during the daytime were calculated by subtracting the preferred temperature at ZT1-ZT3 from the preferred temperature at the peak ( $w^{1118}: \mathrm{ZT} 10-\mathrm{ZT12}$; Pdf $\left.f^{01}: \mathrm{ZT7}-\mathrm{ZT} 9\right) . t$ test: $p=0.3354$. Two-way ANOVA was performed, and there was no significant interaction between $w^{1118}$ and $P d f^{01}$ flies during the daytime $\left(F_{(1,88)}=2.669, p=0.1059\right)$ in Table 4. C, Comparison of preferred temperature decreases at night-onset (ZT10 -ZT12 to $\mathrm{ZT} 13-\mathrm{ZT} 15)$ for $w^{1118}$ and $P d f^{01}$ flies. The values for temperature decreases at night-onset were calculated by subtracting the preferred temperature at ZT13-ZT15 from the preferred temperature at ZT10-ZT12. $t$ test: $p=0.1461$. Two-way ANOVA was performed and there was no significant interaction between $w^{1118}$ and $P d f^{01}$ flies at night-onset $\left(F_{(1,62)}=0.8354, p=0.3643\right)$ in Table 4. D, TPR phenotype of $w^{1118}$ (black line) and Dh3 $37^{\# 51}$ (red line) flies in LD cycles. Error bars indicate the SEM. Italicized numbers represent the number of assays. One-way ANOVA: $p=0.0103$ $\left(F_{(3,34)}=4.386\right)$. Tukey-Kramer test compared with ZT1-ZT3: ${ }^{* * *} p<0.0001$ or ${ }^{* *} p<0.01$ in Table 3.E, Comparison of preferred temperature increases during the daytime (ZT1-ZT12) for $w^{1118}$ and $D h 37^{\# 51}$ flies. The values for temperature increases during the daytime were calculated by subtracting the preferred temperature at ZT1-ZT3 from the preferred temperature at ZT10 -ZT12. $t$ test: $p=0.1080$. Two-way ANOVA was performed and there was no significant interaction between $w^{1118}$ and Dh3 $1^{\# 51}$ flies during the daytime $\left(F_{(1,89)}=1.256, p=0.2653\right)$ in Table 4. $\boldsymbol{F}$, Comparison of preferred temperature decreases at night-onset (ZT10-ZT12 to ZT13-ZT15) for $w^{1118}$ and Dh3 $7^{\sharp 51}$ flies. The values for temperature decreases at night-onset were calculated by subtracting the preferred temperature at ZT13-ZT15 from the preferred temperature at ZT10-ZT12.t test: $p<0.0001$. Two-way ANOVA was performed and there was a significant interaction between $w^{1118}$ and $D h 31^{\# 51}$ flies at night-onset $\left(F_{(1,62)}=6.922, p=0.0107\right)$ in Table 4 .

However, when $P d f r^{5304}$ mutants were backcrossed with $w^{1118}$ $\left(P d f r^{5304-B C}\right)$, they preferred a similar temperature to WT and no longer showed the advanced peak in daytime TPR (Fig. $2 G)$. This suggests that the mild warmer temperature preference and the phase-advanced phenotype of $P d f r^{5304}$ mutants were likely caused by the genetic background. Most importantly, $P d f r^{5304-B C}$ showed a TPR during the daytime similar to that of WT (Fig. $2 \mathrm{H}$ ) and sustained the abnormal night-onset TPR (Fig. 2I).
PDF is not required for night-onset TPR

PDF is a ligand of PDFR that has important roles in circadian rhythms (Renn et al., 1999; Nitabach and Taghert, 2008; Taghert and Nitabach, 2012). We therefore examined the TPR phenotype of $P d f^{01}$. We found that $P d f^{01}$ mutants exhibited a phase-advanced phenotype with a peak at ZT7-ZT9 (Fig. 3A; see Table 3). However, the preferred temperature of $P d f^{01}$ mutants still increased during the daytime (Fig. 3B) and decreased normally at night-onset (Fig. 3C). This suggests that PDF is not required for night-onset TPR. 
A

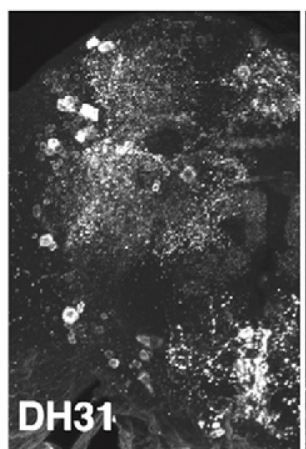

$D h 31^{\# 51}$

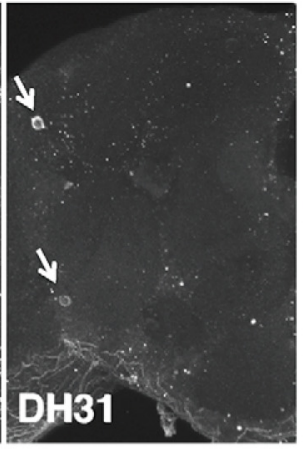

D

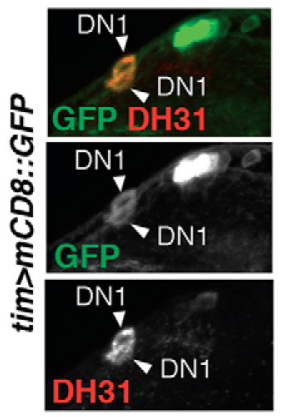

$\mathbf{F}$
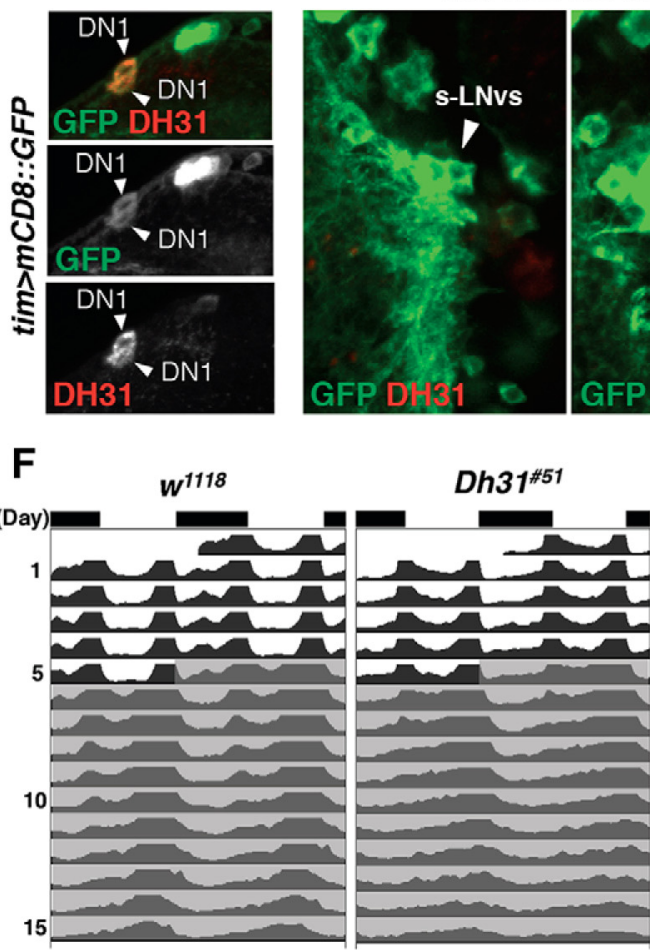

B
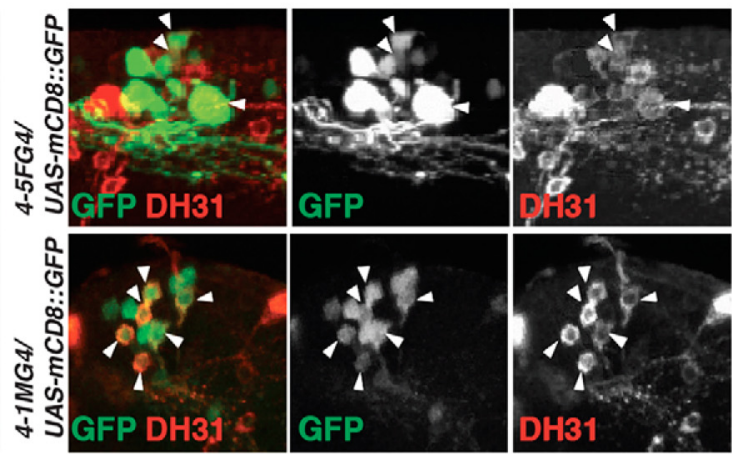

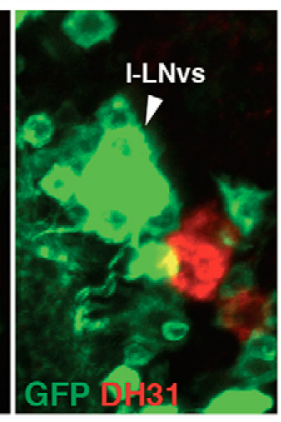

E $\quad$ tim>mCD8::GFP

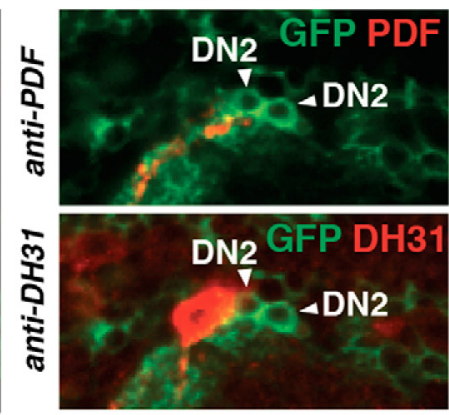

G

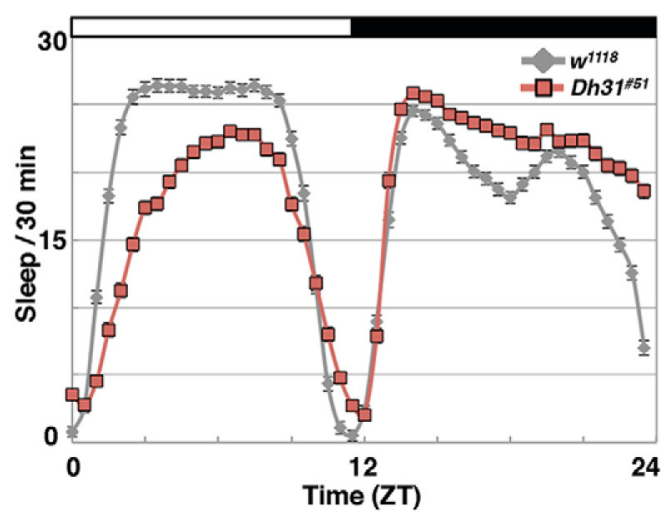

Figure 4. DH31 immunostaining, locomotor activity rhythms, and sleep phenotypes of Dh3 $37^{\# 51}$ flies. A, Immunostaining with anti-DH31 antibody on $w^{1118}$ and $D h 37^{\# 51}$ brains. The images are confocal stacks. Because the epitope regions of the DH31 antibody were deleted in the Dh3 $1^{\# 51}$ mutant (Kunst et al., 2014), the signals (arrows) in Dh31 ${ }^{\# 51}$ brain should be nonspecific signals. B, C, DH31 expression in DN1s (arrowhead) was labeled using Clk4.5F-Gal4 or Clk4.1M-Gal4 (B) or tim-Gal4 (C) with UAS-mCD8:GFP (green) and anti-DH31 antibody (red). $\boldsymbol{D}, \boldsymbol{E}$, No DH31 expression in LNvs or DN2s (arrowhead) was observed using tim-Gal4 with UAS-mCD8:GFP (green) and anti-PDF or anti-DH31 antibodies (red). $\boldsymbol{F}$, Averaged locomotor activity rhythms of $w^{1118}$ and $D h 31^{\# 51}$ flies are shown in a double-plotted actograms. The activity was monitored in LD 12:12 for $5 \mathrm{~d}$ and subsequently in constant darkness for $10 \mathrm{~d}$. $\mathbf{G}$, Sleep profiles for days $2-5$ in LD were calculated for $w^{1118}$ and $D h 31^{\# 51}$ flies.

Additionally, we observed that $P d f^{01}$ mutants preferred a lower temperature during ZT16-ZT24 (Fig. 3A). We noted that PDF-expressing neurons contribute to this lower temperature preference behavior especially before dawn (ZT22ZT24; X. Tang, F.N. Hamada, unpublished observations). Therefore, PDF is likely involved in this lower temperature preference during ZT16-ZT24. On the other hand, since $P d f^{01}$ mutants exhibit normal night-onset TPR, PDFR appears to use other ligands to control night-onset TPR.

\section{DH31 is required for night-onset TPR}

Because PDFR is also activated by DH31 (Mertens et al., 2005), we used a Dh31 mutant, $D h 31^{\# 51}$ in which the DH31 active peptide region was ablated (Head et al., 2015). Using an anti-
Table 1. Comparison of the free-running period and power value between $w^{1118}$ and $D h 31^{\# 51}$ flies

\begin{tabular}{|c|c|c|c|c|c|c|}
\hline \multirow[b]{2}{*}{ Genotype } & \multirow[b]{2}{*}{$n$} & \multirow[b]{2}{*}{$n$ Rhythmic (\%) } & \multicolumn{2}{|c|}{ Tau (hour) } & \multicolumn{2}{|l|}{ Power } \\
\hline & & & Average & SEM & Average & SEM \\
\hline$w^{1118}$ & 118 & 114 (97) & 24.0 & 0.02 & 1371.7 & 75.1 \\
\hline$D h 31^{\# 51}$ & 86 & $80(93)$ & 24.4 & 0.06 & 678.2 & 46.0 \\
\hline
\end{tabular}

body against the active peptide region of DH31 (Kunst et al., 2014), we found that there was no positive signal in the Dh31 $1^{\# 1}$ mutant brain (Fig. 4A). In the WT brain, DH31 was expressed in many cells, including a subset of DN1s labeled by Clk4.5F-Gal4, Clk4.1M-Gal4 (Fig. 4B) and tim-Gal4 (Fig. 4C), but not in other clock cells, such as LNvs or DN2s (Fig. $4 D, E$ ). 


\section{A night-onset}

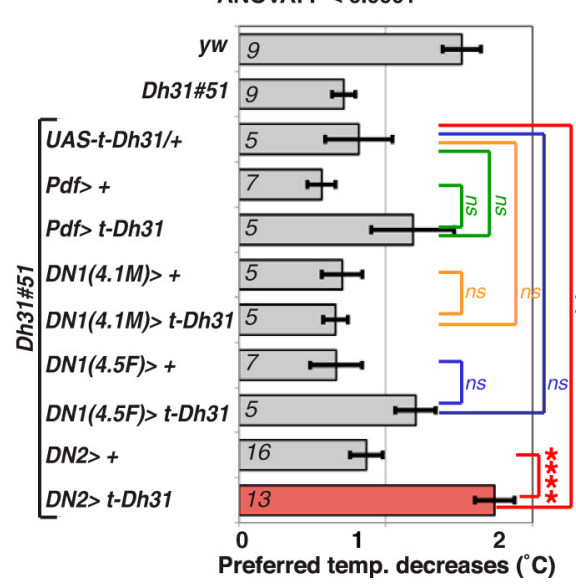

B night-onset

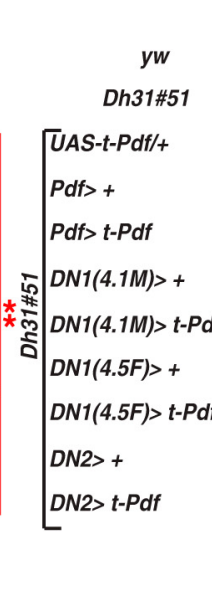

ANOVA: $\mathbf{P}<0.0001$

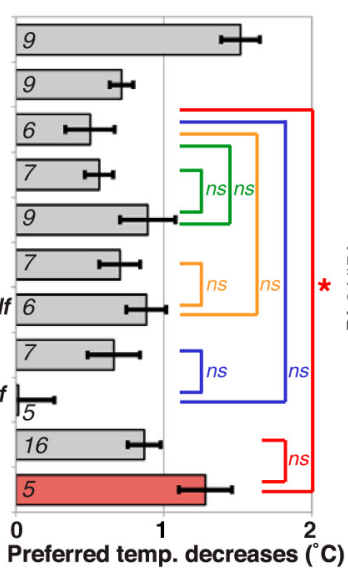

Cnight-onset

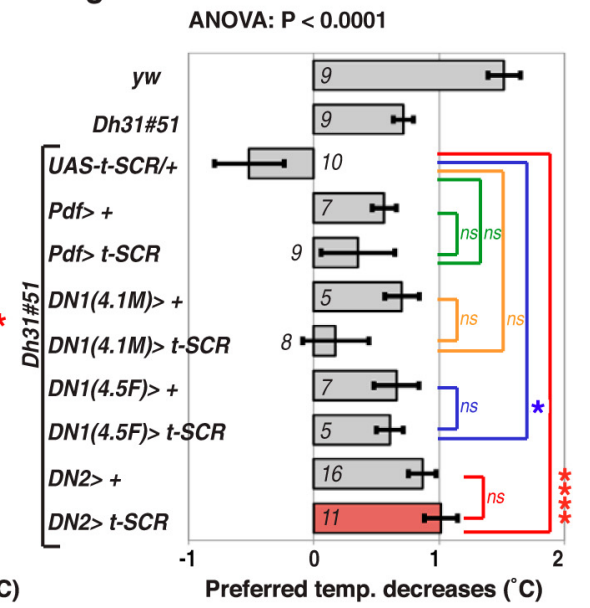

Figure 5. $\quad \mathrm{t}-\mathrm{DH} 31$ expression in DN2s restores night-onset TPR. $A-C$, Comparison of preferred temperature decreases at night-onset (ZT10 -ZT12 to ZT13-ZT15) in Dh37 $7^{\# 51}$ flies with several rescue constructs. UAS-t-Dh31 (A), UAS-t-Pdf (B), or UAS-t-SCR (C) (Choi et al., 2009) was expressed in a subset of the clock cells using the following Gal4 drivers: Pdf (Pdf-Gal4) in s-LNvs and I-LNvs, DN1 (4.1M) (Clk4.1M-Gal4) or DN1 (4.5F) (Clk4.5F-Gal4) in DN1s, and DN2 (Clk9M-Gal4;Pdf-Gal80) in DN2s. Kruskal-Wallis test one-way ANOVA on ranks or one-way ANOVA and posthoc test were used to compare rescue against each control: ${ }^{* * *} p<0.0001,{ }^{* *} p<0.01$, or ${ }^{*} p<0.05$. The detailed statistical analysis is shown in Table 2.

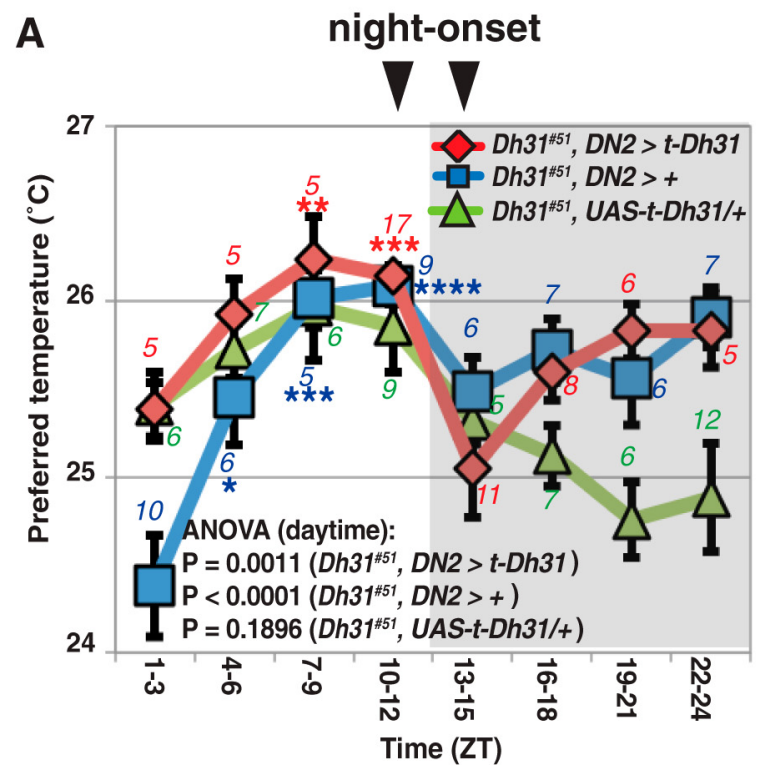

B

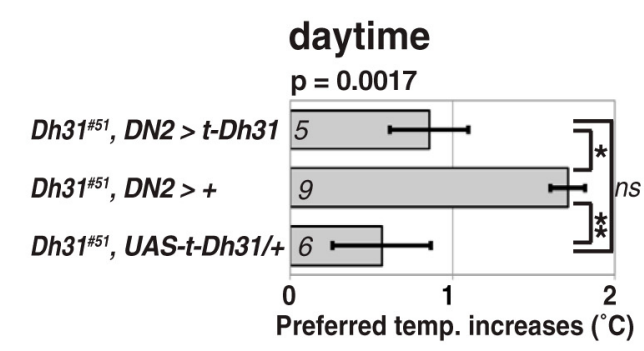

C

\section{night-onset}

$p=0.0069$

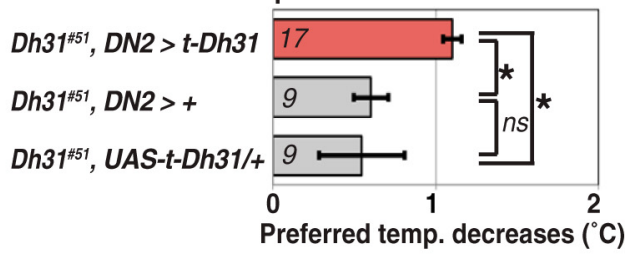

Figure 6. A $24 \mathrm{~h}$ profile of temperature preference behaviors of Dh31 mutant rescue flies. $A$, TPR phenotype of $D h 37^{\# 51}$ rescue flies in DN2s (red: Dh3 $\left.7^{\# 51} ; D N 2>t-D h 31\right)$ and their controls (blue: Dh31 $1^{\# 51}$; DN2 > + or green: Dh31 $1^{\# 51}$; UAS-t-Dh31/+) in LD cycles. UAS-t-Dh31 was expressed in DN2 cells using Clk9M-Gal4, Pdf-Gal80. Error bars indicate the SEM. Italicized numbers represent the number of assays. One-way ANOVA and Tukey-Kramer test were used to compare temperature change based on ZT1-ZT3: ${ }^{* * * *} p<0.0001$, ${ }^{* * *} p<$ $0.001,{ }^{* *} p<0.01$, and ${ }^{*} p<0.05$. The detailed statistical analysis is shown in Table 3. B, Comparison of preferred temperature increases during the daytime (ZT1-ZT12) for Dh3 $1^{\# 51}$ rescue flies. Error bars indicate the SEM. One-way ANOVA and Tukey-Kramer test were used to compare the rescue with each control: ${ }^{* *} p<0.01$ and ${ }^{*} p<0.05$ (Table 2). C, Comparison of preferred temperature decreases at night-onset (ZT10 -ZT12 to ZT13-ZT15) for Dh3 $7^{\# 51}$ rescue flies. Error bars indicate the SEM. One-way ANOVA and Tukey-Kramer test were used to compare the rescue with each control: ${ }^{*} p<0.05$ (Table 2).

A similar pattern of DH31 expression was also shown in a recent study (Kunst et al., 2014). We also examined the sleep and locomotor activity rhythms of $D h 31^{\# 51}$ mutants. Although $D h 31^{\# 51}$ mutants had a relatively higher level of late-night sleep (ZT21-ZT24), they maintained robust locomotor activity rhythms in both the LD and constant dark conditions with a similar period length to WT flies (Fig. 4F, G; Table 1), suggesting that DH31 is involved in sleep regulation but not in circadian rhythmicity. These data were consistent with the results using another Dh31 mutant shown in a previous study (Kunst et al., 2014).
To determine whether DH31 is responsible for TPR, we tested the TPR behavior of $D h 31^{\# 51}$ mutants and found that they exhibited a normal preferred temperature increase during the daytime similar to that of WT flies (Fig. $3 D, E$; see Table 3). However, $D h 31^{\# 51}$ mutants interestingly showed only a $\sim 0.6-0.8^{\circ} \mathrm{C}$ decrease in preferred temperature at night-onset (Fig. $3 F$ ), indicating that DH31 is required for night-onset TPR.

t-DH31 expression in DN2s is sufficient for night-onset TPR Previous studies using imaging analyses have shown that a bath application ofDH31 can activate LNvs (Shafer et al., 2008). To examine 
Table 2. One-way ANOVA analysis of preferred temperature increases during the daytime (ZT1-ZT12) or preferred temperature decreases at night-onset (ZT10-ZT12 and ZT13-ZT15)

\begin{tabular}{|c|c|c|}
\hline Figure & $p$ value & Tests \\
\hline \multicolumn{3}{|l|}{10} \\
\hline$P d f r^{5304} ; P d f>P d f r$ & & \\
\hline $\begin{array}{c}\text { Compared with } \\
\text { Pdfr }{ }^{5304} ; \text { Pdf }>+ \\
\text { Pdfr }{ }^{5304} ; \text { Pdf }>\text { Pdfr }\end{array}$ & NS & $\begin{array}{c}\text { Passed Shapiro-Wilk normality test; } \\
\text { one-way ANOVA: } p=0.1182 ; \\
F_{(\mathrm{DFn}, \mathrm{DFd})}: F_{(2,15)}=2.471\end{array}$ \\
\hline
\end{tabular}

Pdfr ${ }^{5304} ;$ UAS-Pdfr $/+$

Pdfr ${ }^{5304} ;$ UAS-Pdfr/+

Compared with

Pdfr ${ }^{5304} ;$ Pdf $>+$

10

Pdfr ${ }^{504} ;$ DN1(4.5F) $>$ Pdfr

Compared with Pdfr ${ }^{5304} ;$ DN1 (4.5F) $>+$

Pdfr ${ }^{5304} ;$ DN1(4.5F) $>$ Pdfr

Compared with Pdfr ${ }^{5304} ;$ UAS-Pdfr/ +

Pdfr ${ }^{5304}$; UAS-Pdfr/+

Compared with

Pdfr ${ }^{5304} ;$ DN1(4.5F) > +

10

Pdfr ${ }^{5304} ;$ DN2 $>$ Pdfr
Compared with

Pdfr ${ }^{5304} ;$ DN2 $>+$

Pdfr ${ }^{5304}$; DN2 $>$ Pdfr

Compared with

Pdfr ${ }^{5304} ;$ UAS-Pdfr/+

Pdfr ${ }^{5304}$; UAS-Pdfr/+

Compared with

Pdfr ${ }^{5304} ;$ DN2 $>+$

10

Pdfr ${ }^{5304}$; UAS-Pdfr/+

Compared with

Pdfr ${ }^{5304} ;$ Pdf $>+$

Pdfr ${ }^{5304}$; UAS-Pdfrl+

Compared with Pdfr ${ }^{5304} ;$ DN1(4.5F) $>+$

Pdfr ${ }^{5304}$; UAS-Pdfr/+

Compared with

Pdfr ${ }^{5304} ;$ DN2 $>+$

Pdfr ${ }^{5304} ;$ Pdf $>+$

Compared with

Pdfr ${ }^{5304} ;$ DN1(4.5F) $>+$

Pdfr ${ }^{5304} ;$ Pdf $>+$

Compared with

Pdfr ${ }^{5304} ;$ DN2 $>+$

Pdfr ${ }^{5304}$;DN1(4.5F)> +

Compared with

Pdfr ${ }^{5304 ; D N 2>+}$

20

Pdfr ${ }^{5304} ;$ DN2 $>$ Pdfr

Compared with Pdfr ${ }^{5304}$; DN2 $>+$

Pdfr ${ }^{5304} ;$ DN2 $>$ Pdfr

Compared with Pdfr ${ }^{5304}$; UAS-Pdfr/ +

Pdfr ${ }^{5304}$; UAS-Pdfr/+

Compared with

Pdfr ${ }^{5304} ;$ DN2 $>+$

one-way ANOVA: $p=0.0030$;

$F_{(\mathrm{DFn}, \mathrm{DFd})}: F_{(2,14)}=9.066$

NS

Passed Shapiro-Wilk normality test; one-way ANOVA: $p=0.2464$;

$F_{(\mathrm{DFn}, \mathrm{DFd})}: F_{(3,18)}=1.508$

NS

Passed Shapiro-Wilk normality test; one-way ANOVA: $p=0.0169$;

$F_{(\mathrm{DFn}, \mathrm{DFd})}: F_{(2,35)}=4.593$

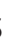

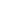

Table 2. Continued

\begin{tabular}{|c|c|c|}
\hline Figure & $p$ value & Tests \\
\hline \multicolumn{3}{|l|}{$2 E$} \\
\hline \multicolumn{3}{|l|}{ Pdfr ${ }^{5304} ;$ DN2 $>$ Pdfr } \\
\hline $\begin{array}{l}\text { Compared with } \\
\text { Pdfr }{ }^{5304} ; \text { DN2>+ }\end{array}$ & $* * *$ & $\begin{array}{l}\text { Passed Shapiro-Wilk normality test; } \\
\text { one-way ANOVA: } p<0.0001 \text {; }\end{array}$ \\
\hline $\begin{array}{l}\text { Pdfr } 5304 ; \text { DN2 }>\text { Pdfr } \\
\text { Compared with }\end{array}$ & $* * * *$ & $F_{(\mathrm{DFn}, \mathrm{DFd})}: F_{(2,35)}=15.32$ \\
\hline $\begin{array}{l}\text { Pdfr }{ }^{5304} ; \text { UAS-Pdfr/ }+ \\
\text { Pdfr }{ }^{5304} ; \text { UAS-Pdfr/ }+\end{array}$ & & \\
\hline $\begin{array}{l}\text { Compared with } \\
\text { Pdfr }{ }^{3304} ; \text { DN2 }>+\end{array}$ & NS & \\
\hline \multicolumn{3}{|l|}{ (2) } \\
\hline$D h 31^{\# 51} ; P d f>t-D h 31$ & & \\
\hline $\begin{array}{l}\text { Compared with } \\
\qquad \text { Dh } 31^{\# 51} ; \text { Pdf }>+\end{array}$ & NS & Kruskal-Wallis test: $p=0.1101$ \\
\hline$D h 31^{\# 51} ; P d f>t-D h 31$ & & \\
\hline $\begin{array}{l}\text { Compared with } \\
\text { Dh } 31^{\# 51} ; \text { UAS-t-Dh31/+ }\end{array}$ & NS & \\
\hline Dh31 ${ }^{\# 51} ;$ UAS-t-Dh31/+ & & \\
\hline $\begin{array}{l}\text { Compared with } \\
\qquad D h 31^{\# 51} ; \text { Pdf }>+\end{array}$ & NS & \\
\hline $5 A$ & & \\
\hline $\begin{array}{l}\text { Dh3 } 31^{\# 51} ; \text { DN1 }(4.1 M)>t-D h 31 \\
\text { Compared with } \\
\text { Dh31 } 31^{\# 51} ; \text { DN1 }(4.1 M)>+ \\
\text { Dh31 } \# 51 ; \text { DN1(4.1M) }>t-D h 31\end{array}$ & NS & $\begin{array}{c}\text { Passed Shapiro-Wilk normality test; } \\
\text { one-way ANOVA: } p=0.7916 ; \\
F_{(\mathrm{DFn}, \mathrm{DFd})} \cdot F_{(2,14)}=0.2377\end{array}$ \\
\hline
\end{tabular}

Compared with

Dh3 $31^{\# 51} ;$ UAS-t-Dh31/+

Dh31 ${ }^{\# 51} ;$ UAS-t-Dh31/+

Compared with

Dh31 $1^{\# 51} ; \operatorname{DN1}(4.1 M)>+$

$5 A$

Dh3 $1^{\# 51} ;$ DN1(4.5F) $>t-D h 31$

Compared with Dh3 $37^{\# 51} ;$ DN1 (4.5F) > +

$D h 31^{\# 51} ; \mathrm{DN1}$ (4.5F) $>t$-Dh31

Compared with Dh31 ${ }^{\# 51} ;$ UAS-t-Dh31/+

Dh31 ${ }^{\# 51}$; UAS-t-Dh31/+

Compared with Dh31 $1^{\# 51} ;$ DN1(4.5F) > +

$5 A$

Dh31 $1^{\# 51} ; D N 2>t-D h 31$

Compared with

$D h 33^{\# 51} ; D N 2>+$

Dh3 $3^{\# 51} ; D N 2>t-D h 31$

Compared with

Dh31 151 $^{*}$ UAS-t-Dh31/+

Dh37"51;UAS-t-Dh31/+

Compared with

Dh $31^{\# 51} ;$ DN2 $>+$

$5 A$

Dh31" ${ }^{\# 51} ;$ UAS-t-Dh31/+

Compared with Dh3 $37^{\# 51} ;$ Pdf $>+$

Dh31 ${ }^{\# 51} ;$ UAS-t-Dh31/+

Compared with Dh31 $1^{\# 51} ;$ DN1 $(4.1 M)>+$

Dh31 ${ }^{\# 51}$; UAS-t-Dh31/+

Compared with Dh31" ${ }^{\# 1} ;$ DN1(4.5F) $>+$
NS

NS

NS

NS

NS

NS

NS
Passed Shapiro-Wilk normality test; one-way ANOVA: $p=0.1446$;

$F_{(\mathrm{DFn}, \mathrm{DFd})}: F_{(2,14)}=2.228$ 
Table 2. Continued

\begin{tabular}{|c|c|c|}
\hline Figure & $p$ value & Tests \\
\hline $\begin{array}{l}\text { Dh3 } 31^{\# 51} ; \text { UAS-t-Dh31/+ } \\
\text { Compared with } \\
\text { Dh3 } 31^{\# 51} ; \text { DN2>+ }\end{array}$ & NS & \\
\hline $\begin{array}{l}\text { Compared with } \\
\text { Dh31 }{ }^{\# 51} ; \text { DN1 }(4.1 M)>+ \\
\text { Dh3 } 37^{\# 51} ; \text { Pdf }>+\end{array}$ & NS & \\
\hline 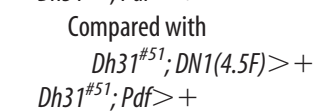 & NS & \\
\hline $\begin{array}{c}\text { Compared with } \\
\text { Dh31 } 31^{\# 51} ; \text { DN2 }>+ \\
D h 37^{\# 51} ; D N 1(4.1 M)>+\end{array}$ & NS & \\
\hline 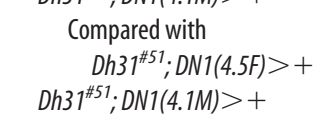 & NS & \\
\hline 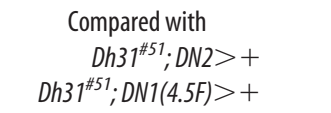 & NS & \\
\hline $\begin{array}{l}\text { Compared with } \\
\qquad \text { Dh } 31^{\# 51} ; D N 2>+\end{array}$ & NS & \\
\hline \multicolumn{3}{|l|}{$5 B$} \\
\hline \multicolumn{3}{|l|}{$D h 31^{\# 51} ;$ Pdf $>$ t-Pdf } \\
\hline $\begin{array}{c}\text { Compared with } \\
\text { Dh31 } 351 ; P d f>+ \\
\text { Dh31 }{ }^{\# 51} ; P d f>t-P d f\end{array}$ & NS & $\begin{array}{c}\text { Passed Shapiro-Wilk normality test; } \\
\text { one-way ANOVA: } p=0.1975 ; \\
F_{(\mathrm{DFn}, \mathrm{DFd})}: F_{(2,19)}=1.769\end{array}$ \\
\hline $\begin{array}{c}\text { Compared with } \\
\text { Dh31 } 351 ; \text { UAS-t-Pdf/+ } \\
\text { Dh31 } 1^{\# 51} ; \text { UAS-t-Pdf/+ }\end{array}$ & NS & \\
\hline $\begin{array}{l}\text { Compared with } \\
\text { Dh3 } 31^{\# 51} ; \text { Pdf }>+\end{array}$ & NS & \\
\hline \multicolumn{3}{|l|}{$5 B$} \\
\hline $\begin{array}{l}\text { Dh3 } 37^{\# 51} ; \text { DN1 }(4.1 M)>t-P d f \\
\text { Compared with } \\
\text { Dh31 } \\
\text { Dh31 } ; \text { DN1 } 14.1 M)>+ \\
\text { \#51 } D N 1(4.1 M)>t-P d f\end{array}$ & NS & $\begin{array}{l}\text { Passed Shapiro-Wilk normality test; } \\
\text { one-way ANOVA: } p=0.7238 ; \\
F_{\text {int }}:\end{array}$ \\
\hline $\begin{array}{l}\text { Compared with } \\
\text { Dh3 } 3 \text { t }^{\# 1} ; \text { UAS-t-Pdf/+ } \\
\text { Dh3 } 31^{\# 51} ; \text { UAS-t-Pdf/+ }\end{array}$ & NS & \\
\hline $\begin{array}{l}\text { Compared with } \\
\qquad \text { Dh3 } 31^{\# 51} ; \text { DN1(4.1M) >+ }\end{array}$ & NS & \\
\hline \multicolumn{3}{|l|}{$5 B$} \\
\hline 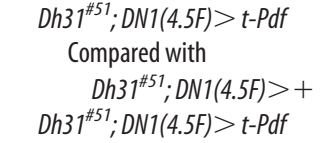 & NS & $\begin{array}{l}\text { Passed Kolmogorov-Smirnov test } \\
\text { with Dallal-Wilkinson-Lillie for } \\
\text { p value; one-way ANOVA: }\end{array}$ \\
\hline 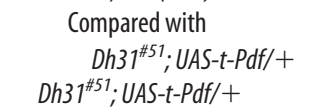 & NS & $\begin{array}{l}p=0.0924 ; F_{(\mathrm{DFn}, \mathrm{DFd})} \\
F_{(2,15)}=2.803\end{array}$ \\
\hline $\begin{array}{l}\text { Compared with } \\
\text { Dh31 } 1^{\# 51} ; \text { DN1(4.5F) }>+\end{array}$ & NS & \\
\hline \multicolumn{3}{|l|}{ (1) } \\
\hline $\begin{array}{c}\text { Compared with } \\
\text { Dh3 } 31^{\# 51} ; \text { DN2 }>+ \\
\text { Dh3 } 31^{\# 51} ; \text { DN2 }>t-P d f\end{array}$ & NS & $\begin{array}{l}\text { Passed Shapiro-Wilk normality test; } \\
\text { one-way ANOVA: } p=0.0226 ; \\
F_{(\mathrm{DFn}, \mathrm{DFd})}: F_{(2,24)}=4.454\end{array}$ \\
\hline $\begin{array}{c}\text { Compared with } \\
\text { Dh31 } 1^{\# 51} ; \text { UAS-t-Pdf/+ } \\
\text { Dh31 }{ }^{\# 51} ; \text { UAS-t-Pdf/+ }\end{array}$ & * & \\
\hline $\begin{array}{l}\text { Compared with } \\
\text { Dh } 31^{\# 51} ; \text { DN2 }>+\end{array}$ & NS & \\
\hline
\end{tabular}

Table 2. Continued

\begin{tabular}{|c|c|c|}
\hline Figure & $p$ value & Tests \\
\hline \multicolumn{3}{|l|}{$5 B$} \\
\hline \multicolumn{3}{|l|}{ Dh31" ${ }^{\# 51} ;$ UAS-t-Pdf/+ } \\
\hline 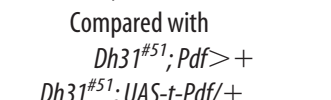 & NS & $\begin{array}{l}\text { Passed Shapiro-Wilk normality test } \\
\text { one-way ANOVA: } p=0.2378 \text {; }\end{array}$ \\
\hline 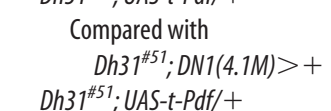 & NS & \\
\hline 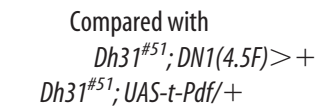 & NS & \\
\hline 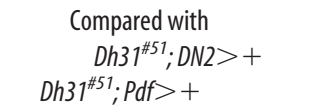 & NS & \\
\hline 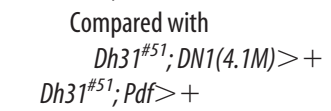 & NS & \\
\hline $\begin{array}{c}\text { Compared with } \\
\text { Dh3 } 31^{\# 51} ; \text { DN1(4.5F) }>+ \\
D h 31^{\# 51} ; \text { Pdf }>+\end{array}$ & NS & \\
\hline $\begin{array}{c}\text { Compared with } \\
\text { Dh31 } 1^{\# 51} ; \text { DN2 }>+ \\
\text { Dh31 }{ }^{\# 51} ; \text { DN1 }(4.1 M)>+\end{array}$ & NS & \\
\hline 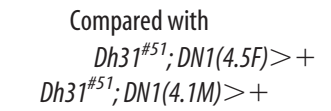 & NS & \\
\hline $\begin{array}{l}\text { Compared with } \\
\text { Dh } 31^{\# 51} ; \text { DN2 }>+\end{array}$ & NS & \\
\hline $\begin{array}{c}\text { Dh31 } 1^{\# 51} ; \text { DN1 }(4.5 F)>+ \\
\text { Compared with } \\
\text { Dh3 } 31^{\# 51} ; \text { DN2>+ }\end{array}$ & NS & \\
\hline 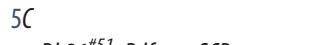 & & \\
\hline
\end{tabular}

Passed Shapiro-Wilk normality test; Compared with Dh3 $31^{\# 51} ; P d f>+$ $D h 37^{\# 51} ;$ Pdf $>t-S C R$ Compared with Dh3 $31^{\# 51} ;$ UAS-t-SCR/+ Dh3 $7^{\# 51} ;$ UAS-t-SCR/+ Compared with Dh3 $31^{\# 51} ;$ Pdf $>+$

$5 C$

Dh31 $1^{\# 51} ;$ DN1 $\left.4.1 M\right)>t-S C R$ Compared with Dh31 ${ }^{\# 51} ; \operatorname{DN1}(4.1 M)>+$ Dh3 $7^{\# 51} ;$ DN1 $(4.1 M)>t-S C R$ Compared with Dh31 ${ }^{\# 51}$; UAS-t-SCR/+ Dh37 $7^{\not 51} ;$ UAS-t-SCR/+ Compared with Dh31 $1^{\# 51} ;$ DN1(4.1M)> +

$5 C$

Dh3 $37^{\# 51} ; D N 1(4.5 F)>t-S C R$

Compared with Dh31 $1^{\# 51} ;$ DN1(4.5F) $>+$

Dh3 $31^{\# 51} ; D N 1(4.5 F)>t-S C R$ Compared with Dh31 ${ }^{\# 51}$;UAS-t-SCR/+ one-way ANOVA: $p=0.0166$; $F_{(\mathrm{DFn}, \mathrm{DFd})}: F_{(2,23)}=4.924$ Passed Shapiro-Wilk normality test; one-way ANOVA: $p=0.0092$; $F_{(\mathrm{DFn}, \mathrm{DFd})}: F_{(2,22)}=5.849$

Passed Shapiro-Wilk normality test; one-way ANOVA: $p=0.0030$; $F_{(\mathrm{DFn}, \mathrm{DFd})}: F_{(2,19)}=7.997$

(Table Continues) 
Table 2. Continued

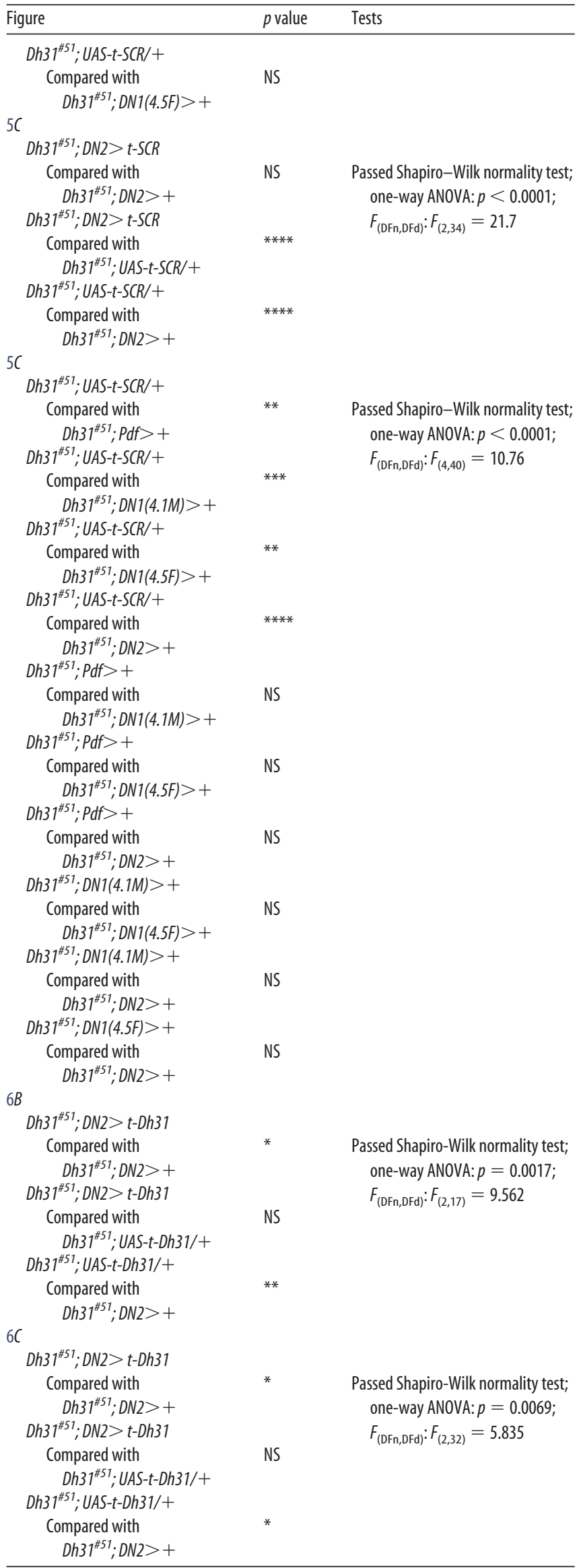

whether DH31 acts on LNvs to regulate night-onset TPR, we used t-DH31 (Choi et al., 2009, 2012). This membrane-tethered peptide has both linker and anchor peptides that couple with the cell membrane, resulting in cell-autonomous binding and activation of its receptors on specific cells (Choi et al., 2009, 2012).

First, we expressed t-DH31 in LNvs using Pdf-Gal4 in the Dh31 ${ }^{\# 51}$ background and tested night-onset TPR (Fig. 5A). Although the decrease in the preferred temperature of rescue flies was slightly greater than decreases for Gal4 and UAS controls, the decrease was not statistically significant (Fig. 5A, green; Table 2). This suggests that DH31 acting on LNvs is not sufficient for night-onset TPR. Furthermore, a recent study has shown that DN1s secrete DH31 (Kunst et al., 2014). Because neuropeptides can function in autocrine signaling (Im and Taghert, 2010), we expressed t-DH31 in a subset of DN1s using Clk4.1M-Gal4 or Clk4.5F-Gal4 in the Dh31 $1^{\# 5}$ background. However, the decreases of preferred temperature in these flies were not statistically different from those of their control flies (Fig. 5A, orange and blue; Table 2). Therefore, the data suggest that DH31 acting on DN1s is also not sufficient for night-onset TPR.

Since we showed that PDFR expression in DN2s is important for night-onset TPR (Fig. 1D), we hypothesized that DH31 might function as a ligand of PDFR for night-onset TPR. If so, DH31 may act on DN2s through PDFR to control preferred temperature decreases. To examine this, t-DH31 was expressed in DN2s using Clk9M-Gal4; Pdf-Gal80 (Figs. 5A, 6) in the Dh31 ${ }^{\# 51}$ background. The flies displayed restored night-onset TPR, in which the preferred temperature decreases of these rescue flies were statistically different from those of Gal4 and UAS controls (Figs. $5 A$, red, $6 A, C$; Tables 2, 3). This indicates that t-DH31 expression in DN2s is sufficient for night-onset TPR.

To examine the specificity of t-DH31 expression in DN2s, $\mathrm{t}-\mathrm{PDF}$ or scrambled PDF (t-SCR) was expressed in DN2s in the Dh31 $1^{\# 51}$ background as controls (Fig. 5B,C). Although t-PDF expression in DN2s slightly rescued night-onset TPR compared with UAS controls (UAS-t-Pdfl+ in $D h 31^{\# 51}$; Fig. $5 B$, red), the preferred temperature decreases were not statistically different from those of the Gal4 control flies (Clk9M-Gal4; Pdf-Gal80 in $D h 31^{\# 51}$; Table 2). Additionally, we observed that the control flies using t-SCR did not restore their night-onset TPR (Fig. 5C). Thus, the data indicate that DH31 acting on DN2s is critical for night-onset TPR.

It should be noted that flies with t-DH31 (both DN2s rescue and UAS control flies) showed a smaller preferred temperature increases than the flies without t-DH31 (Gal4 control flies) during the daytime (Fig. $6 A, B$ ). It is possible that the UAS-t-Dh31 transgene itself or t-DH31 leaky expression might prevent a normal preferred temperature increase during the daytime. Nonetheless, with regard to nightonset (ZT10-ZT12 to ZT13-ZT15; Table 4), only t-DH31 expression in DN2s could rescue the Dh31 mutant phenotype (Fig. $5 A$ ). Also, t-PDF and t-SCR in DN2s did not restore night-onset TPR (Fig. $5 B, C$ ). Therefore, we concluded that t-DH31 expression in DN2s is sufficient for regulating night-onset TPR.

\section{Discussion}

We showed that both $D h 31$ and $P d f r$ mutants exhibited abnormal night-onset TPR and that t-DH31 and PDFR expression in DN2s are sufficient to control night-onset TPR, suggesting that DH31 could be a ligand of PDFR in DN2s (Fig. 7B). Unexpectedly, PDF, an important neuropeptide for locomotor activity, is not required for night-onset TPR. It suggests that PDF and DH31 appear to act on different subsets of PDFR-expressing clock cells to regulate locomotor activity 
Table 3. One-way ANOVA analysis to compare TPR at different times of the day

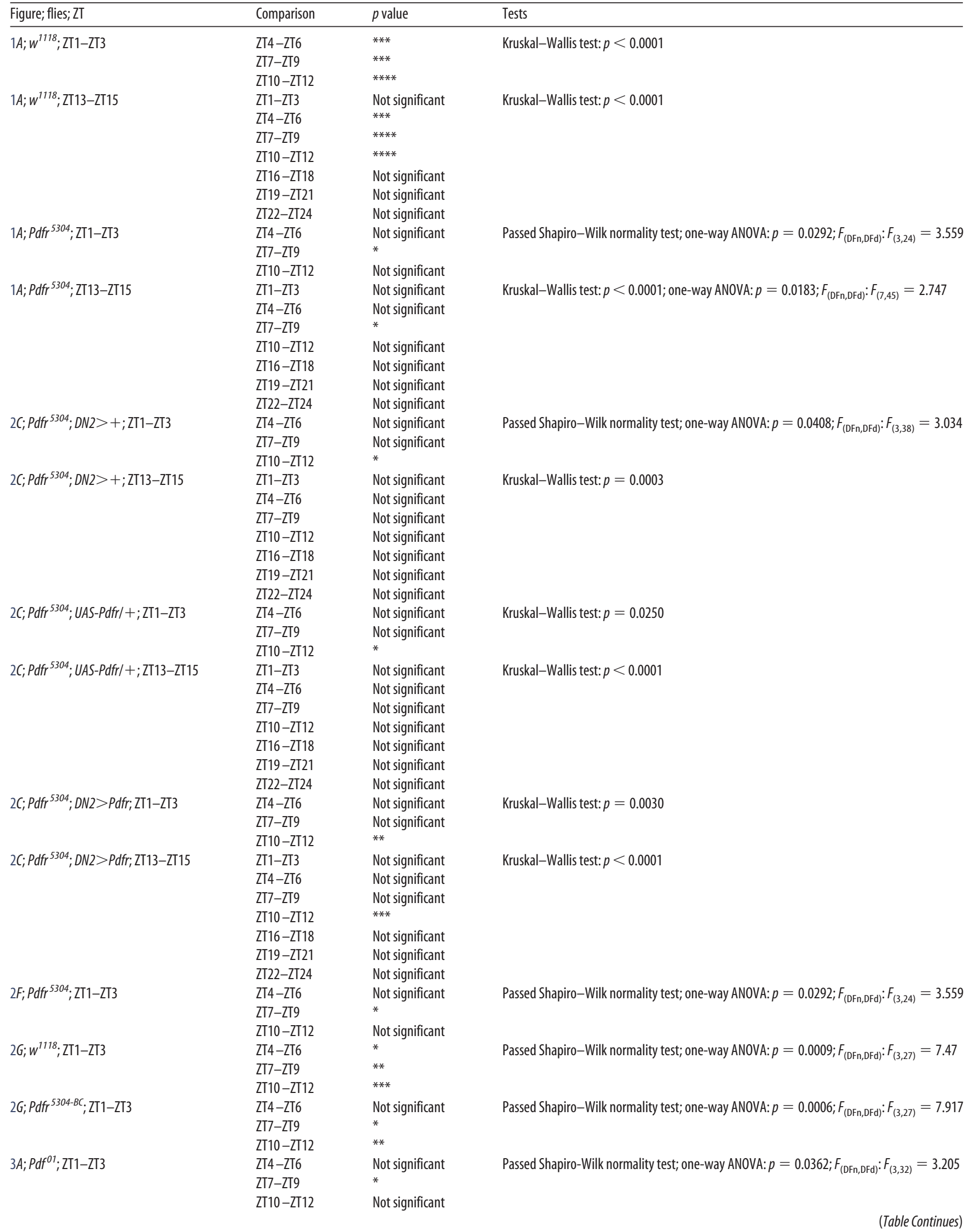


Table 3. Continued

\begin{tabular}{|c|c|c|c|}
\hline Figure; flies; ZT & Comparison & $p$ value & Tests \\
\hline $3 A ; P d f^{01} ; \mathrm{ZT} 13-\mathrm{ZT} 15$ & $\begin{array}{l}\text { ZT1-ZT3 } \\
\text { ZT4-ZT6 } \\
\text { ZT7-ZT9 } \\
\text { ZT10-ZT12 } \\
\text { ZT16-ZT18 } \\
\text { ZT19-ZT21 } \\
\text { ZT22-ZT24 }\end{array}$ & $\begin{array}{l}\text { Not significant } \\
\text { Not significant } \\
* \\
\text { Not significant } \\
\text { Not significant } \\
\text { Not significant } \\
\text { Not significant }\end{array}$ & Kruskal-Wallis test: $p<0.0001$ \\
\hline 3D;Dh31 ${ }^{\# 51} ; \mathrm{ZT} 1-\mathrm{ZT} 3$ & $\begin{array}{l}\text { ZT4-ZT6 } \\
\text { ZT7-ZT9 } \\
\text { ZT10-ZT12 }\end{array}$ & $\begin{array}{l}\text { Not significant } \\
\text { Not significant } \\
\text { ** }\end{array}$ & Passed Shapiro-Wilk normality test; one-way ANOVA: $p=0.0103 ; F_{(\mathrm{DFn}, \mathrm{DFd})}: F_{(3,34)}=4.386$ \\
\hline 3D;Dh37 ${ }^{\# 51} ; Z \mathrm{ZT} 13-Z T 15$ & $\begin{array}{l}\text { ZT1-ZT3 } \\
\text { ZT4-ZT6 } \\
\text { ZT7-ZT9 } \\
\text { ZT10-ZT12 } \\
\text { ZT16-ZT18 } \\
\text { ZT19-ZT21 } \\
\text { ZT22-ZT24 }\end{array}$ & $\begin{array}{l}\text { Not significant } \\
\text { Not significant } \\
\text { Not significant } \\
\text { Not significant } \\
\text { Not significant } \\
\text { Not significant } \\
\text { Not significant }\end{array}$ & Kruskal-Wallis test: $p=0.1072$ \\
\hline $6 A ; D h 31^{\# 51} ; D N 2>t-D h 31 ; Z T 1-Z T 3$ & $\begin{array}{l}\text { ZT4-ZT6 } \\
\text { ZT7-ZT9 } \\
\text { ZT10-ZT12 }\end{array}$ & $\begin{array}{l}\text { Not significant } \\
* * \\
* * *\end{array}$ & Passed Shapiro-Wilk normality test; one-way ANOVA: $p=0.0011 ; F_{(\mathrm{DFn}, \mathrm{DFd})}: F_{(3,28)}=7.087$ \\
\hline $6 A ; D h 31^{\# 51} ; D N 2>t-D h 31 ; Z T 13-Z T 15$ & $\begin{array}{l}\text { ZT1-ZT3 } \\
\text { ZT4-ZT6 } \\
\text { ZT7-ZT9 } \\
\text { ZT10-ZT12 } \\
\text { ZT16-ZT18 } \\
\text { ZT19-ZT21 } \\
\text { ZT22-ZT24 }\end{array}$ & $\begin{array}{l}\text { Not significant } \\
\text { Not significant } \\
* * \\
\text { **** } \\
\text { Not significant } \\
\text { Not significant } \\
\text { Not significant }\end{array}$ & Passed Shapiro-Wilk normality test; one-way ANOVA: $p=0.0001 ; F_{(\mathrm{DFn}, \mathrm{DFd})}: F_{(7,54)}=5.392$ \\
\hline $6 A ; D h 31^{\# 51} ;$ DN2 $>+; Z T 1-Z T 3$ & $\begin{array}{l}\text { ZT4-ZT6 } \\
\text { ZT7-ZT9 } \\
\text { ZT10-ZT12 }\end{array}$ & $\begin{array}{l}* \\
* * * \\
* * * *\end{array}$ & Passed Shapiro-Wilk normality test; one-way ANOVA: $p<0.0001 ; F_{(\mathrm{DFn}, \mathrm{DFd})}: F_{(3,26)}=12.9$ \\
\hline $6 A ; D h 31^{\# 51} ;$ DN2 $>+; Z T 13-Z T 15$ & $\begin{array}{l}\text { ZT1-ZT3 } \\
\text { ZT4-ZT6 } \\
\text { ZT7-ZT9 } \\
\text { ZT10-ZT12 } \\
\text { ZT16-ZT18 } \\
\text { ZT19-ZT21 } \\
\text { ZT22-ZT24 }\end{array}$ & $\begin{array}{l}* \\
\text { Not significant } \\
\text { Not significant } \\
\text { Not significant } \\
\text { Not significant } \\
\text { Not significant } \\
\text { Not significant }\end{array}$ & Passed Shapiro-Wilk normality test; one-way ANOVA: $p<0.0001 ; F_{(\mathrm{DFn}, \mathrm{DFd})}: F_{(7,48)}=7.449$ \\
\hline $6 A ;$ Dh31 ${ }^{\# 51} ;$ UAS-t-Dh31/+; ZT1-ZT3 & $\begin{array}{l}\text { ZT4-ZT6 } \\
\text { ZT7-ZT9 } \\
\text { ZT10-ZT12 }\end{array}$ & $\begin{array}{l}\text { Not significant } \\
\text { Not significant } \\
\text { Not significant }\end{array}$ & Kruskal-Wallis test: $p=0.1896$ \\
\hline $6 A ;$ Dh31 ${ }^{\# 51} ;$ UAS-t-Dh31/+;ZT13-ZT15 & $\begin{array}{l}\text { ZT1-ZT3 } \\
\text { ZT4-ZT6 } \\
\text { ZT7-ZT9 } \\
\text { ZT10-ZT12 } \\
\text { ZT16-ZT18 } \\
\text { ZT19-ZT21 } \\
\text { ZT22-ZT24 }\end{array}$ & $\begin{array}{l}\text { Not significant } \\
\text { Not significant } \\
\text { Not significant } \\
\text { Not significant } \\
\text { Not significant } \\
\text { Not significant } \\
\text { Not significant }\end{array}$ & Kruskal-Wallis test: $p=0.0108$ \\
\hline
\end{tabular}

rhythms and night-onset TPR, respectively (Fig. $7 A, B$ ). Together, our data suggest that locomotor activity and nightonset TPR are regulated through PDFR by different subsets of clock neurons using different neuropeptides (Fig. 7A,B). Nonetheless, because all Dh31, Pdf, and Pdfr mutants still maintain normal daytime TPR (Figs. 1, 2, 3), the underlying molecular mechanisms of daytime TPR are still obscure.

In humans, body temperature dramatically decreases at night, which is associated with sleep initiation (Gilbert et al., 2004; Kräuchi, 2007a,b). Although it suggests a relationship between BTR and sleep-wake cycles, the underlying mechanisms are largely unclear. A recent study suggested that DH31 mediates sleep-wake cycles and that DH31 secretion functions as a wakepromoting signal before dawn (Kunst et al., 2014) (Fig. 7C). Given that we found that DH31 is required for night-onset TPR,
DH31 could regulate both sleep-wake cycles and TPR with different timing (i.e., at night-onset for TPR and before dawn for sleep; Fig. $7 B, C$ ).

While t-DH31 expression in DN2s rescues the Dh31 ${ }^{\# 51}$ phenotype (Fig. 5A), t-PDF expression in DN2s slightly rescues in comparison with UAS controls (UAS-t-Pdfl+; Fig. $5 B$ ). This shows that $\mathrm{t}-\mathrm{DH} 31$ in $\mathrm{DN} 2 \mathrm{~s}$ rescues more efficiently than $\mathrm{t}-\mathrm{PDF}$ in DN2s. However, DH31 stimulates PDFR less efficiently than PDF in vitro (Mertens et al., 2005). This suggests that either DH31 may more efficiently bind PDFR in DN2s than PDF or that DN2s may express another receptor of DH31 that also regulates night-onset TPR. Notably, although the $\mathrm{DH} 31$ receptor $(\mathrm{DH} 31 \mathrm{R})$ is a known receptor for $\mathrm{DH} 31$ in vitro, we have observed that $\mathrm{DH} 31 \mathrm{R}$ is not expressed in DN2s and that the Dh31r mutant exhibited normal night-onset TPR (T. Goda, F.N. Hamada, unpublished observations). Therefore, it 
Table 4. Two-way ANOVA analysis to compare the preferred temperature at ZT1-ZT3 and ZT10 -ZT12 as well as ZT10 -ZT12 and ZT13-ZT15

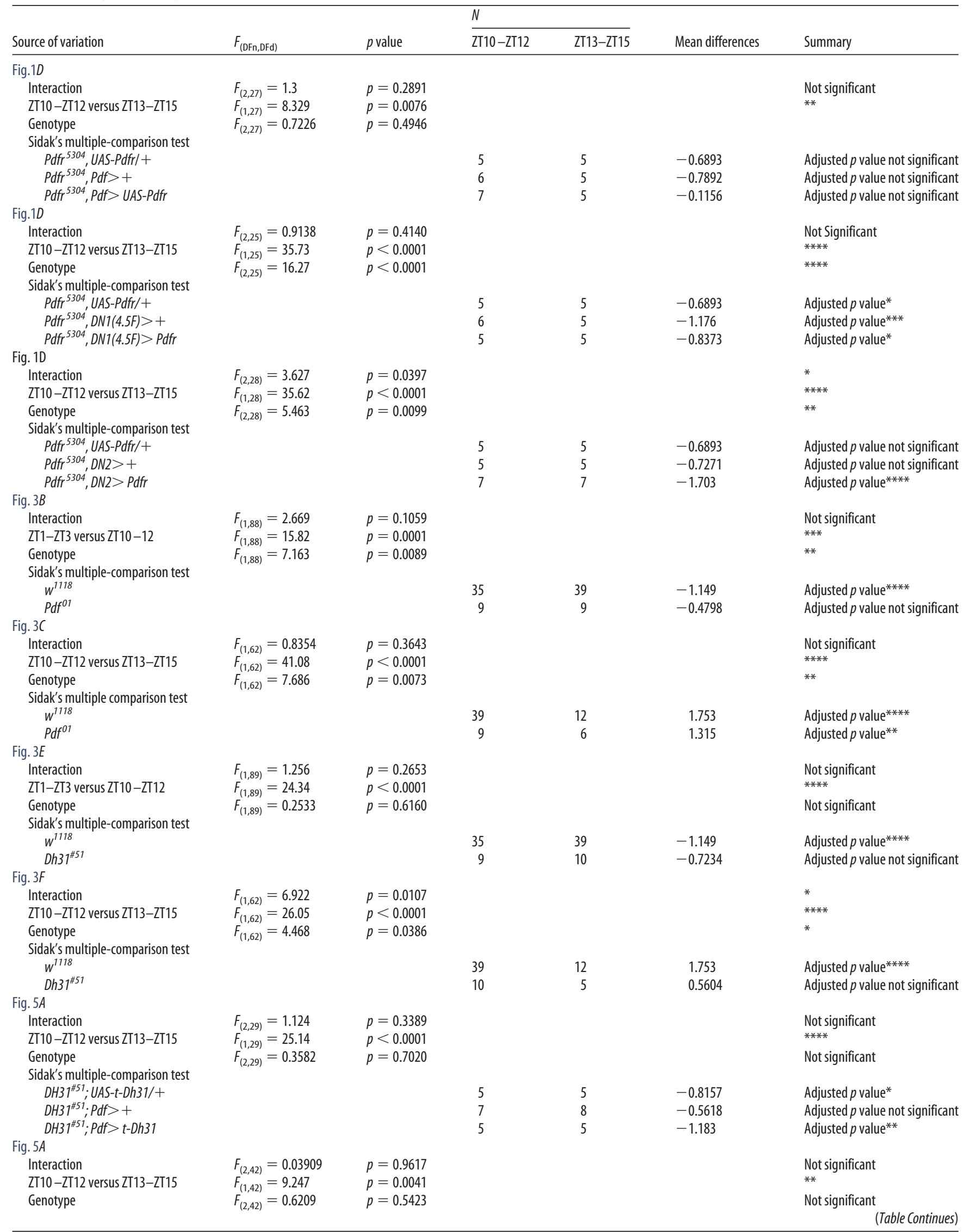


Table 4. Continued

\begin{tabular}{|c|c|c|c|c|c|c|}
\hline \multirow[b]{2}{*}{ Source of variation } & \multirow[b]{2}{*}{$F_{(\mathrm{DFn}, \mathrm{DFd})}$} & \multirow[b]{2}{*}{$p$ value } & \multicolumn{2}{|l|}{$N$} & \multirow[b]{2}{*}{ Mean differences } & \multirow[b]{2}{*}{ Summary } \\
\hline & & & ZT10-ZT12 & ZT13-ZT15 & & \\
\hline \multicolumn{7}{|l|}{ Sidak's multiple-comparison test } \\
\hline DH31 $1^{\# 51} ;$ UAS-t-Dh31/+ & & & 5 & 5 & -0.8157 & Adjusted $p$ value not significant \\
\hline$D H 31^{\# 51} ; D N 1(4.1 M)>+$ & & & 7 & 21 & -0.7021 & Adjusted $p$ value not significant \\
\hline$D H 31^{\# 51} ; D N 1(4.1 M)>t-D h 31$ & & & 5 & 5 & -0.6573 & Adjusted $p$ value not significant \\
\hline \multicolumn{7}{|l|}{ Fig. $5 A$} \\
\hline Interaction & $F_{(2,28)}=0.803$ & $p=0.4580$ & & & & Not significant \\
\hline ZT10 -ZT12 versus ZT13-ZT15 & $F_{(1,28)}=24.61$ & $p<0.0001$ & & & & $* * * *$ \\
\hline Genotype & $F_{(2,28)}=1.457$ & $p=0.2501$ & & & & Not significant \\
\hline \multicolumn{7}{|l|}{ Sidak's multiple-comparison test } \\
\hline DH31 ${ }^{\# 51} ;$ UAS-t-Dh31/+ & & & 5 & 5 & -0.8157 & Adjusted $p$ value not significant \\
\hline$D H 31^{\# 51} ; D N 1(4.5 F)>+$ & & & 7 & 5 & -0.6604 & Adjusted $p$ value not significant \\
\hline$D H 31^{\# 51} ; D N 1(4.5 F)>t-D h 31$ & & & 5 & 7 & -1.203 & Adjusted $p$ value ${ }^{* *}$ \\
\hline \multicolumn{7}{|l|}{ Fig. $5 A$} \\
\hline Interaction & $F_{(2,48)}=4.433$ & $p=0.0171$ & & & & * \\
\hline ZT10 -ZT12 versus ZT13-ZT15 & $F_{(1,48)}=64.06$ & $p<0.0001$ & & & & $* * * *$ \\
\hline Genotype & $F_{(2,48)}=5.161$ & $p=0.0093$ & & & & $* *$ \\
\hline \multicolumn{7}{|l|}{ Sidak's multiple-comparison test } \\
\hline DH31 ${ }^{\# 51} ;$ UAS-t-Dh31/+ & & & 5 & 5 & -0.8157 & Adjusted $p$ value* \\
\hline$D H 31^{\# 51} ; D N 2>+$ & & & 16 & 7 & -0.8666 & Adjusted $p$ value ${ }^{* * *}$ \\
\hline$D H 31^{\# 51} ; D N 2>t-D h 31$ & & & 13 & 8 & -1.741 & Adjusted $p$ value $e^{* * * *}$ \\
\hline
\end{tabular}

would be interesting to further explore whether additional receptors for $\mathrm{DH} 31$ in DN2s are involved in night-onset TPR.

We previously showed that flies in the light prefer a $1^{\circ} \mathrm{C}$ higher temperature than in the dark (light-dependent temperature preference [LDTP]) (Head et al., 2015) (Fig. 7D), thus demonstrating that light influences temperature preference behavior. Importantly, while LDTP is only affected by light, night-onset TPR is affected by both light and time (ZT10-ZT12 and ZT13-ZT15). Therefore, they are not controlled by the same pathway (Fig. $7 C, D)$. In fact, we showed that PDFR expression using Clk4.5F-Gal4 rescues the abnormal LDTP phenotype of $P d f r^{5304}$ mutants (Head et al., 2015), but it did not rescue night-onset TPR (Fig. $1 D$, blue).

DH31 is a functional homolog of mammalian CGRP (Johnson et al., 2005). Importantly, CGRP is related to many physiological functions, such as temperature sensation, migraines, and chronic pain in mammals (Arulmani et al., 2004; Tsujikawa et al., 2007; Benemei et al., 2009; Seybold, 2009). CGRP has recently attracted attention for its role in the treatment of migraine attacks, which are associated with bodytemperature fluctuations (Durham and Vause, 2010; Ordás et al., 2013) and correlate with the time of day (Fox and Davis, 1998). Additionally, CGRP is expressed in the SCN, but its function in circadian rhythms is not entirely clear (Park et al., 1993). Thus, our findings raise the possibility that CGRP may be involved in BTR regulation and may mediate the connection between the circadian clock and other physiological functions. Moreover, this research will not only expand our necessary and sufficient for LDTP.
A B
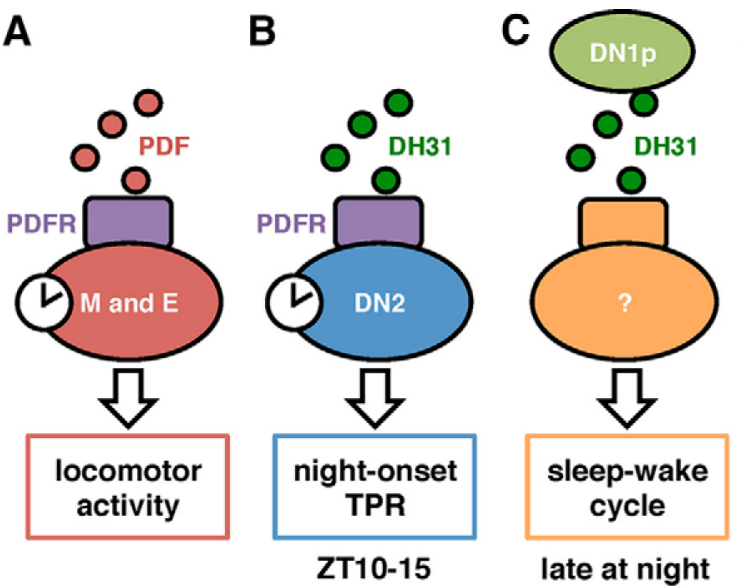

D

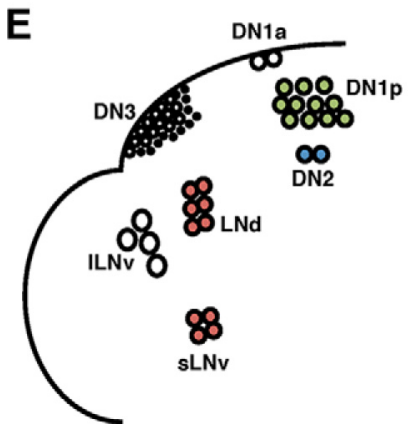

late at night

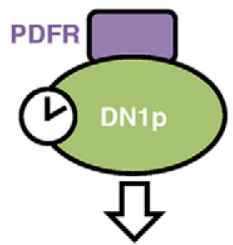

light dependent

temp preference (LDTP)

Figure 7. $\boldsymbol{A}-\boldsymbol{E}$, Schematic summaries of locomotor activity $(A)$, night-onset TPR (B), sleep (C), light dependent temperature preference (LDTP) (D), and clock cells in the brain (E). $A$, PDF binds to PDFR in M and E cells to regulate locomotor activity. $B, D H 31$ binds to PDFR in DN2s to regulate night-onset TPR. C, DH31 expression in DN1ps mediates sleep-wake cycles. D, PDFR in DN1ps are

current understanding of neuropeptidergic regulation, but may also ultimately facilitate a discovery of the fundamental mechanisms of BTR.

\section{References}

Arulmani U, Maassenvandenbrink A, Villalón CM, Saxena PR (2004) Calcitonin gene-related peptide and its role in migraine pathophysiology. Eur J Pharmacol 500:315-330. CrossRef Medline 
Aschoff J (1983) Circadian control of body temperature. J Therm Biol 8:143-147.

Aton SJ, Colwell CS, Harmar AJ, Waschek J, Herzog ED (2005) Vasoactive intestinal polypeptide mediates circadian rhythmicity and synchrony in mammalian clock neurons. Nat Neurosci 8:476-483. Medline

Benemei S, Nicoletti P, Capone JG, Geppetti P (2009) CGRP receptors in the control of pain and inflammation. Curr Opin Pharmacol 9:9-14. CrossRef Medline

Brown SA, Zumbrunn G, Fleury-Olela F, Preitner N, Schibler U (2002) Rhythms of mammalian body temperature can sustain peripheral circadian clocks. Curr Biol 12:1574-1583. CrossRef Medline

Buhr ED, Yoo SH, Takahashi JS (2010) Temperature as a universal resetting cue for mammalian circadian oscillators. Science 330:379-385. CrossRef Medline

Choi C, Fortin JP, McCarthy EV, Oksman L, Kopin AS, Nitabach MN (2009) Cellular dissection of circadian peptide signals with genetically encoded membrane-tethered ligands. Curr Biol 19:1167-1175. CrossRef Medline

Choi C, Cao G, Tanenhaus AK, McCarthy EV, Jung M, Schleyer W, Shang Y, Rosbash M, Yin JC, Nitabach MN (2012) Autoreceptor control of peptide/neurotransmitter corelease from PDF neurons determines allocation of circadian activity in drosophila. Cell Rep 2:332-344. CrossRef Medline

Coast GM, Webster SG, Schegg KM, Tobe SS, Schooley DA (2001) The Drosophila melanogaster homologue of an insect calcitonin-like diuretic peptide stimulates V-ATPase activity in fruit fly Malpighian tubules. J Exp Biol 204:1795-1804. Medline

Dubruille R, Emery P (2008) A plastic clock: how circadian rhythms respond to environmental cues in Drosophila. Mol Neurobiol 38:129-145. CrossRef Medline

Durham PL, Vause CV (2010) Calcitonin gene-related peptide (CGRP) receptor antagonists in the treatment of migraine. CNS Drugs 24:539-548. CrossRef Medline

Duvall LB, Taghert PH (2012) The circadian neuropeptide PDF signals preferentially through a specific adenylate cyclase isoform AC3 in M pacemakers of Drosophila. PLoS Biol 10:e1001337. CrossRef Medline

Fox AW, Davis RL (1998) Migraine chronobiology. Headache 38:436-441. CrossRef Medline

Gerhart-Hines Z, Feng D, Emmett MJ, Everett LJ, Loro E, Briggs ER, Bugge A, Hou C, Ferrara C, Seale P, Pryma DA, Khurana TS, Lazar MA (2013) The nuclear receptor Rev-erbalpha controls circadian thermogenic plasticity. Nature 503:410-413. CrossRef Medline

Gilbert SS, van den Heuvel CJ, Ferguson SA, Dawson D (2004) Thermoregulation as a sleep signalling system. Sleep Med Rev 8:81-93. CrossRef Medline

Glossop NR, Houl JH, Zheng H, Ng FS, Dudek SM, Hardin PE (2003) VRILLE feeds back to control circadian transcription of clock in the Drosophila circadian oscillator. Neuron 37:249-261. CrossRef Medline

Goda T, Leslie JR, Hamada FN (2014) Design and analysis of temperature preference behavior and its circadian rhythm in Drosophila. J Vis Exp 83:e51097. CrossRef Medline

Hamada FN, Rosenzweig M, Kang K, Pulver SR, Ghezzi A, Jegla TJ, Garrity PA (2008) An internal thermal sensor controlling temperature preference in Drosophila. Nature 454:217-220. CrossRef Medline

Hannibal J, Hsiung HM, Fahrenkrug J (2011) Temporal phasing of locomotor activity, heart rate rhythmicity, and core body temperature is disrupted in VIP receptor 2-deficient mice. Am J Physiol Regul Integr Comp Physiol 300:R519-R530. CrossRef Medline

Harmar AJ, Marston HM, Shen S, Spratt C, West KM, Sheward WJ, Morrison CF, Dorin JR, Piggins HD, Reubi JC, Kelly JS, Maywood ES, Hastings MH (2002) The VPAC(2) receptor is essential for circadian function in the mouse suprachiasmatic nuclei. Cell 109:497-508. CrossRef Medline

Head LM, Tang X, Hayley SE, Goda T, Umezaki Y, Chang EC, Leslie JR, Fujiwara M, Garrity PA, Hamada FN (2015) The influence of light on temperature preference in Drosophila. Curr Biol 25:1063-1068. CrossRef Medline

Hyun S, Lee Y, Hong ST, Bang S, Paik D, Kang J, Shin J, Lee J, Jeon K, Hwang S, Bae E, Kim J (2005) Drosophila GPCR Han is a receptor for the circadian clock neuropeptide PDF. Neuron 48:267-278. CrossRef Medline

Im SH, Taghert PH (2010) PDF receptor expression reveals direct interactions between circadian oscillators in Drosophila. J Comp Neurol 518: 1925-1945. CrossRef Medline

Johnson EC, Shafer OT, Trigg JS, Park J, Schooley DA, Dow JA, Taghert PH (2005) A novel diuretic hormone receptor in Drosophila: evidence for conservation of CGRP signaling. J Exp Biol 208:1239-1246. CrossRef Medline

Kaneko H, Head LM, Ling J, Tang X, Liu Y, Hardin PE, Emery P, Hamada FN (2012) Circadian rhythm of temperature preference and its neural control in Drosophila. Curr Biol 22:1851-1857. CrossRef Medline

Kräuchi K (2002) How is the circadian rhythm of core body temperature regulated? Clin Auton Res 12:147-149. CrossRef Medline

Kräuchi K (2007a) The human sleep-wake cycle reconsidered from a thermoregulatory point of view. Physiol Behav 90:236-245. CrossRef Medline

Kräuchi K (2007b) The thermophysiological cascade leading to sleep initiation in relation to phase of entrainment. Sleep Med Rev 11:439-451. CrossRef Medline

Kunst M, Hughes ME, Raccuglia D, Felix M, Li M, Barnett G, Duah J, Nitabach MN (2014) Calcitonin gene-related peptide neurons mediate sleep-specific circadian output in Drosophila. Curr Biol 24:2652-2664. CrossRef Medline

Lear BC, Merrill CE, Lin JM, Schroeder A, Zhang L, Allada R (2005) A G-protein-coupled receptor, groom-of-PDF, is required for PDF neuron action in circadian behavior. Neuron 48:221-227. CrossRef Medline

McCoy ES, Taylor-Blake B, Street SE, Pribisko AL, Zheng J, Zylka MJ (2013) Peptidergic CGRPalpha primary sensory neurons encode heat and itch and tonically suppress sensitivity to cold. Neuron 78:138-151. CrossRef Medline

Mertens I, Vandingenen A, Johnson EC, Shafer OT, Li W, Trigg JS, De Loof A, Schoofs L, Taghert PH (2005) PDF receptor signaling in Drosophila contributes to both circadian and geotactic behaviors. Neuron 48:213219. CrossRef Medline

Nitabach MN, Taghert PH (2008) Organization of the Drosophila circadian control circuit. Curr Biol 18:R84-R93. CrossRef Medline

Ordás CM, Cuadrado ML, Rodríguez-Cambrón AB, Casas-Limón J, del Prado N, Porta-Etessam J (2013) Increase in body temperature during migraine attacks. Pain Med 14:1260-1264. CrossRef Medline

Park HT, Baek SY, Kim BS, Kim JB, Kim JJ (1993) Calcitonin gene-related peptide-like immunoreactive (CGRPI) elements in the circadian system of the mouse: an immunohistochemistry combined with retrograde transport study. Brain Res 629:335-341. CrossRef Medline

Refinetti R (1999) Relationship between the daily rhythms of locomotor activity and body temperature in eight mammalian species. Am J Physiol 277:R1493-R1500. Medline

Refinetti R, Menaker M (1992) The circadian rhythm of body temperature. Physiol Behav 51:613-637. CrossRef Medline

Renn SC, Park JH, Rosbash M, Hall JC, Taghert PH (1999) A pdf neuropeptide gene mutation and ablation of PDF neurons each cause severe abnormalities of behavioral circadian rhythms in Drosophila. Cell 99:791-802. CrossRef Medline

Saper CB, Lu J, Chou TC, Gooley J (2005a) The hypothalamic integrator for circadian rhythms. Trends Neurosci 28:152-157. CrossRef Medline

Saper CB, Scammell TE, Lu J (2005b) Hypothalamic regulation of sleep and circadian rhythms. Nature 437:1257-1263. CrossRef Medline

Satlin A, Volicer L, Stopa EG, Harper D (1995) Circadian locomotor activity and core-body temperature rhythms in Alzheimer's disease. Neurobiol Aging 16:765-771. CrossRef Medline

Schmid B, Helfrich-FörsterC, Yoshii T (2011) A new ImageJ plug-in "ActogramJ" for chronobiological analyses. J Biol Rhythms 26:464-467. CrossRef Medline

Schroeder A, Loh DH, Jordan MC, Roos KP, Colwell CS (2011) Circadian regulation of cardiovascular function: a role for vasoactive intestinal peptide. Am J Physiol Heart Circ Physiol 300:H241-250. CrossRef Medline

Seybold VS (2009) The role of peptides in central sensitization. Handb Exp Pharmacol 194:451-491. CrossRef Medline

Shafer OT, Kim DJ, Dunbar-Yaffe R, Nikolaev VO, Lohse MJ, Taghert PH (2008) Widespread receptivity to neuropeptide PDF throughout the neuronal circadian clock network of Drosophila revealed by real-time cyclic AMP imaging. Neuron 58:223-237. CrossRef Medline

Sokolove PG, Bushell WN (1978) The chi square periodogram: its utility for analysis of circadian rhythms. J Theor Biol 72:131-160. CrossRef Medline

Stevenson RD (1985) The relative importance of behavioral and physiological adjustments controlling body temperature in terrestrial ectotherms. Am Nat 126:362-386. CrossRef

Stoleru D, Peng Y, Nawathean P, Rosbash M (2005) A resetting signal be- 
tween Drosophila pacemakers synchronizes morning and evening activity. Nature 438:238-242. CrossRef Medline

Taghert PH, Nitabach MN (2012) Peptide neuromodulation in invertebrate model systems. Neuron 76:82-97. CrossRef Medline

Tang X, Platt MD, Lagnese CM, Leslie JR, Hamada FN (2013) Temperature integration at the AC thermosensory neurons in Drosophila. J Neurosci 33:894-901. CrossRef Medline

Tomita J, Mitsuyoshi M, Ueno T, Aso Y, Tanimoto H, Nakai Y, Aigaki T, Kume S, Kume K (2011) Pan-neuronal knockdown of calcineurin reduces sleep in the fruit fly, Drosophila melanogaster. J Neurosci 31:1313713146. CrossRef Medline

Tsujikawa K, Yayama K, Hayashi T, Matsushita H, Yamaguchi T, Shigeno T, Ogitani Y, Hirayama M, Kato T, Fukada S, Takatori S, Kawasaki H, Okamoto H, Ikawa M, Okabe M, Yamamoto H (2007) Hypertension and dysregulated proinflammatory cytokine production in receptor activitymodifying protein 1-deficient mice. Proc Natl Acad Sci U S A 104:1670216707. CrossRef Medline

Umezaki Y, Yoshii T, Kawaguchi T, Helfrich-FörsterC, Tomioka K (2012)
Pigment-dispersing factor is involved in age-dependent rhythm changes in Drosophila melanogaster. J Biol Rhythms 27:423-432. CrossRef Medline

Weinert D (2010) Circadian temperature variation and ageing. Ageing Res Rev 9:51-60. CrossRef Medline

Weinert D, Waterhouse J (1998) Diurnally changing effects of locomotor activity on body temperature in laboratory mice. Physiol Behav 63:837843. CrossRef Medline

Welsh DK, Takahashi JS, Kay SA (2010) Suprachiasmatic nucleus: cell autonomy and network properties. Annu Rev Physiol 72:551-577. CrossRef Medline

Zhang L, Chung BY, Lear BC, Kilman VL, Liu Y, Mahesh G, Meissner RA, Hardin PE, Allada R (2010) DN1(p) circadian neurons coordinate acute light and PDF inputs to produce robust daily behavior in Drosophila. Curr Biol 20:591-599. CrossRef Medline

Zhang Y, Liu Y, Bilodeau-Wentworth D, Hardin PE, Emery P (2010) Light and temperature control the contribution of specific DN1 neurons to Drosophila circadian behavior. Curr Biol 20:600-605. CrossRef Medline 